Proceedings of the Edinburgh Mathematical Society (2003) 46, 293-314 (C)

DOI:10.1017/S0013091502000664 Printed in the United Kingdom

\title{
A UNIQUENESS THEOREM FOR AN INVERSE ELECTROMAGNETIC SCATTERING PROBLEM IN INHOMOGENEOUS ANISOTROPIC MEDIA
}

\author{
FIORALBA CAKONI AND DAVID COLTON \\ Department of Mathematical Sciences, University of Delaware, Newark, \\ DE 19716, USA (cakoni@math.udel.edu; colton@math.udel.edu)
}

(Received 3 July 2002)

\begin{abstract}
We show that the support of a (possibly) coated anisotropic medium is uniquely determined by the electric far-field patterns corresponding to incident time-harmonic electromagnetic plane waves with arbitrary polarization and direction. Our proof avoids the use of a fundamental solution to Maxwell's equations in an anisotropic medium and instead relies on the well-posedness and regularity properties of solutions to an interior transmission problem for Maxwell's equations.

Keywords: anisotropic media; electromagnetic scattering; conductive boundary condition; inverse scattering; mixed boundary conditions; transmission problem

2000 Mathematics subject classification: Primary 35R30; 35Q60

Secondary 35P25; 78A45
\end{abstract}

\section{Introduction}

In the past 10 years considerable progress has been made in the mathematical investigation of the inverse scattering problem for electromagnetic waves [7]. Of central importance in this investigation is the derivation of various uniqueness theorems for the inverse scattering problem at fixed frequency. In particular, given the far-field patterns of scattered time-harmonic electromagnetic waves corresponding to incident plane waves with arbitrary direction and polarization, the question was asked if the shape of an impenetrable scatterer, or the index of refraction of an inhomogeneous penetrable scatterer, can be uniquely determined. In the case of a perfect conductor, this question was answered affirmatively in 1992 [6, Theorem 7.1] by using the ideas of Kirsch and Kress for the case of acoustic scattering [14]. At about the same time it was shown by Colton and Päivärinta $[\mathbf{8}]$ that the index of refraction of an inhomogeneous isotropic medium with constant permeability was uniquely determined by the far-field pattern. This result was subsequently generalized to the case of variable permeability by Ola, Päivärinta and Somersalo [20] and by Ola and Somersalo [19]. Further progress in establishing uniqueness results for inverse electromagnetic scattering problems was made by Hähner [10] and Hettlich [13], who considered transmission problems for isotropic media. Hähner also gave a simplified and improved version of the theorem of Colton and Päivärinta [12]. 
The key tool used in all of the above work was a clever use of fundamental solutions for Maxwell's equations. In the case of obstacle scattering the fundamental solution of concern was the classical dyadic Green function, whereas for the case of an inhomogeneous, isotropic medium a new class of exponentially growing solutions to Maxwell's equations was introduced. Given the success of the above approach, there is considerable interest in extending the above collection of ideas from the case of isotropic media to the anisotropic case. Of course, for anisotropic media standard examples show that the index of refraction (which is now a tensor) is not in fact uniquely determined, but rather what is possible to determine is the support of the inhomogeneity [22]. However, even with this more limited objective, serious problems arise in pursuing the above programme to prove uniqueness for anisotropic media due to the difficulty in constructing and analysing a fundamental solution to Maxwell's equations for anisotropic media without putting overly restrictive conditions on the constitutive parameters. A way around this difficulty was recently proposed for the case of acoustic waves by Hähner [11], who was able to avoid the use of fundamental solutions for elliptic equations with variable coefficients by using regularity results for the solution to what is called an interior transmission problem (cf. [3], [21] and [6, $\S 8.4]$ ). In [11] Hähner says that 'this structure of the proof hopefully turns out to be useful in other inhomogeneous medium problems', and in our view this paper is a confirmation of his hope for a class of problems in electromagnetic scattering theory.

As indicated above, the problem we consider in this paper is to show that the support of a bounded anisotropic scattering object is uniquely determined by the far-field patterns corresponding to electromagnetic plane waves of arbitrary direction and polarization. We are in fact able to consider the more general case when the anisotropic scatterer is possibly partly coated by a thin layer of a highly conductive material, i.e. on part of the boundary the electromagnetic field is allowed to (possibly) satisfy a conductive boundary condition [1]. This problem is of particular interest in the detection of decoys, e.g. wooden tanks coated by metallic paint (with the ultimate aim of distinguishing real tanks from the decoys). We begin our paper by using variational methods to show that the mixed boundary-value problem described above is well posed and sufficiently regular. We then introduce a class of modified interior transmission problems for Maxwell's equations and use the programme of Hähner to arrive at the desired uniqueness theorem. As is usual in going from the case of acoustic waves to electromagnetic waves, the main difficulty occurs in the different spaces needed to analyse the boundary-value problems for Maxwell's equations, in our case the space $X\left(D, \Gamma_{2}\right)$ (cf. $\left.\S 2.2\right)$ instead of the standard Sobolev space $H^{1}(D)$ used in the scalar case.

\section{The direct scattering problem}

\subsection{Formulation of the problem}

Let $D \subset \mathbb{R}^{3}$ be a bounded open set having a $C^{2}$-smooth boundary $\Gamma$ such that the exterior domain $D_{\mathrm{e}}:=\mathbb{R}^{3} \backslash \bar{D}$ is connected. The unit normal vector to $\Gamma$ directed into the exterior of $D$ is denoted by $\nu$. We assume that the boundary $\Gamma=\Gamma_{1} \cup \Pi \cup \Gamma_{2}$ is 
split in two open disjoint parts $\Gamma_{1}$ and $\Gamma_{2}$ having $\Pi$ as their possible common boundary in $\Gamma$. The domain $D$ is the support of an anisotropic (possibly disconnected) object that is partly coated on a portion $\Gamma_{2}$ of the boundary by a very thin layer of a high conductivity material and the incident field is a time-harmonic electromagnetic plane wave with frequency $\omega\left(\Gamma_{2}\right.$ may be the empty set!). The interior electric and magnetic fields, $\tilde{E}^{\text {int }}, \tilde{H}^{\text {int }}$, and the exterior electric and magnetic fields, $\tilde{E}^{\text {ext }}, \tilde{H}^{\text {ext }}$, satisfy

$$
\left.\begin{array}{r}
\nabla \times \tilde{E}^{\mathrm{ext}}-\mathrm{i} \omega \mu_{0} \tilde{H}^{\mathrm{ext}}=0 \\
\nabla \times \tilde{H}^{\mathrm{ext}}+\mathrm{i} \omega \epsilon_{0} \tilde{E}^{\mathrm{ext}}=0
\end{array}\right\} \quad \text { in } D_{\mathrm{e}},
$$

and on the boundary $\Gamma$

$$
\begin{aligned}
& \nu \times \tilde{E}^{\mathrm{ext}}-\nu \times \tilde{E}^{\mathrm{int}}=0 \quad \text { on } \Gamma, \\
& \nu \times \tilde{H}^{\mathrm{ext}}-\nu \times \tilde{H}^{\mathrm{int}}=0 \quad \text { on } \Gamma_{1}, \\
& \nu \times \tilde{H}^{\mathrm{ext}}-\nu \times \tilde{H}^{\mathrm{int}}=\tilde{\eta}(x)\left(\nu \times \tilde{E}^{\mathrm{ext}}\right) \times \nu \quad \text { on } \Gamma_{2} .
\end{aligned}
$$

The electric permittivity $\epsilon_{0}$ and magnetic permeability $\mu_{0}$ of the exterior dielectric medium are positive constants, whereas the scatterer has the same magnetic permeability $\mu_{0}$ as the exterior medium but the electric permittivity $\epsilon$ and conductivity $\sigma$ are real $3 \times 3$-matrix-valued functions. The function $\tilde{\eta}>0$, defined on the portion $\Gamma_{2}$ of the boundary, describes the physical properties of the thin coating layer $[\mathbf{1}]$ and $\omega$ denotes the frequency. If we define

$$
\begin{gathered}
\tilde{E}^{(\text {ext,int })=} \frac{1}{\sqrt{\epsilon_{0}}} E^{(\text {ext,int })}, \quad \tilde{H}^{(\text {ext }, \text { int })}=\frac{1}{\sqrt{\mu_{0}}} H^{(\text {ext }, \text { int })}, \quad k^{2}=\epsilon_{0} \mu_{0} \omega^{2}, \\
n(x)=\frac{1}{\epsilon_{0}}\left(\epsilon(x)+\mathrm{i} \frac{\sigma(x)}{\omega}\right), \quad \tilde{\eta}(x)=\sqrt{\frac{\mu_{0}}{\epsilon_{0}}} \eta(x),
\end{gathered}
$$

we obtain the transmission problem

$$
\begin{aligned}
& \left.\begin{array}{l}
\nabla \times E^{\text {ext }}-\mathrm{i} k H^{\text {ext }}=0 \\
\nabla \times H^{\text {ext }}+\mathrm{i} k E^{\text {ext }}=0
\end{array}\right\} \quad \text { in } D_{\mathrm{e}}, \\
& \left.\begin{array}{r}
\nabla \times E^{\mathrm{int}}-\mathrm{i} k H^{\mathrm{int}}=0 \\
\nabla \times H^{\mathrm{int}}+\mathrm{i} k n(x) E^{\mathrm{int}}=0
\end{array}\right\} \quad \text { in } D, \\
& \nu \times E^{\mathrm{ext}}-\nu \times E^{\mathrm{int}}=0 \quad \text { on } \Gamma, \\
& \nu \times H^{\text {ext }}-\nu \times H^{\text {int }}=0 \quad \text { on } \Gamma_{1}, \\
& \nu \times H^{\mathrm{ext}}-\nu \times H^{\mathrm{int}}=\eta(x)\left(\nu \times E^{\mathrm{ext}}\right) \times \nu \quad \text { on } \Gamma_{2},
\end{aligned}
$$


where the exterior field $E^{\text {ext }}, H^{\text {ext }}$ is given by

$$
\begin{aligned}
& E^{\text {ext }}=E^{\mathrm{i}}+E^{\mathrm{s}}, \\
& H^{\text {ext }}=H^{\mathrm{i}}+H^{\mathrm{s}},
\end{aligned}
$$

$E^{\mathrm{s}}, H^{\mathrm{s}}$ is the scattered field satisfying the Silver-Müller radiation condition

$$
\lim _{r \rightarrow \infty}\left(H^{\mathrm{s}} \times x-r E^{\mathrm{s}}\right)=0
$$

uniformly in $\hat{x}=x /|x|, r=|x|$, the incident field $E^{\mathrm{i}}, H^{\mathrm{i}}$ is given by

$$
\left.\begin{array}{rl}
E^{\mathrm{i}}(x):=\frac{\mathrm{i}}{k} \nabla \times \nabla \times p \mathrm{e}^{\mathrm{i} k x \cdot d} & =\mathrm{i} k(d \times p) \times d \mathrm{e}^{\mathrm{i} k x \cdot d}, \\
H^{\mathrm{i}}(x):=\nabla \times p \mathrm{e}^{\mathrm{i} k x \cdot d}=\mathrm{i} k d \times p \mathrm{e}^{\mathrm{i} k x \cdot d},
\end{array}\right\}
$$

the wavenumber $k$ is positive, $d$ is a unit vector giving the direction of propagation and $p$ is the polarization vector. In the following we assume that $n$ is a $3 \times 3$-matrixvalued function whose entries are $C^{1}(\bar{D})$ functions such that $n$ is symmetric and satisfies $\bar{\xi} \cdot \operatorname{Im}(n) \xi \geqslant 0$ and $\bar{\xi} \cdot \operatorname{Re}(n) \xi \geqslant \gamma|\xi|^{2}$ for all $\xi \in \mathbb{C}^{3}$ and all $x \in \bar{D}$, where $\gamma$ is a positive constant. Finally, we assume that $\eta>0$ is a bounded $C^{1}$ function supported on $\Gamma_{2}$. We again remind the reader that $\Gamma_{2}$ may be the empty set.

\subsection{The direct problem}

In order to formulate precisely the problem we are concerned with throughout this paper, we need the following spaces. Letting $\left(H^{\mathrm{s}}(D)\right)^{3},\left(H_{\text {loc }}^{\mathrm{s}}\left(D_{\mathrm{e}}\right)\right)^{3}$ and $\left(H^{\mathrm{s}}(\Gamma)\right)^{3}, s \in \mathbb{R}$, denote the product of the standard Sobolev spaces defined on $D, D_{\mathrm{e}}$ and $\Gamma$, respectively (with the convention $H^{0}=L^{2}$ ), and

$$
\begin{aligned}
H(\operatorname{curl}, D) & :=\left\{u \in\left(L^{2}(D)\right)^{3}: \nabla \times u \in\left(L^{2}(D)\right)^{3}\right\}, \\
L_{t}^{2}(\Gamma) & :=\left\{u \in\left(L^{2}(\Gamma)\right)^{3}: \nu \cdot u=0 \text { on } \Gamma\right\}, \\
L_{t}^{2}\left(\Gamma_{2}\right) & :=\left\{\left.u\right|_{\Gamma_{2}}: u \in L_{t}^{2}(\Gamma)\right\},
\end{aligned}
$$

we introduce the space

$$
X\left(D, \Gamma_{2}\right):=\left\{u \in H(\operatorname{curl}, D): \nu \times\left. u\right|_{\Gamma_{2}} \in L_{t}^{2}\left(\Gamma_{2}\right)\right\}
$$

equipped with the norm

$$
\|u\|_{X\left(D, \Gamma_{2}\right)}^{2}=\|u\|_{H(\operatorname{curl}, D)}^{2}+\|\nu \times u\|_{L^{2}\left(\Gamma_{2}\right)}^{2} .
$$

For the exterior domain $D_{\mathrm{e}}$ we define the above spaces in the same way for every $D_{\mathrm{e}} \cap B_{R}$, with $B_{R}$ a ball of arbitrary radius $R$ and denote these spaces by $H_{\text {loc }}\left(\operatorname{curl}, D_{\mathrm{e}}\right)$ and $X_{\text {loc }}\left(D_{\mathrm{e}}, \Gamma_{2}\right)$, respectively. The trace spaces of $\nu \times\left. u\right|_{\Gamma}$ and $\nu \times\left.(u \times \nu)\right|_{\Gamma}$ for $u \in H(\operatorname{curl}, D)$ (or $\left.u \in H_{\mathrm{loc}}\left(\operatorname{curl}, D_{\mathrm{e}}\right)\right)$ are given by

$$
\begin{aligned}
& H_{\text {div }}^{-1 / 2}(\Gamma):=\left(u \in\left(H^{-1 / 2}(\Gamma)\right)^{3}, \nu \cdot u=0, \operatorname{div}_{\Gamma} u \in H^{-1 / 2}(\Gamma)\right), \\
& H_{\text {curl }}^{-1 / 2}(\Gamma):=\left(u \in\left(H^{-1 / 2}(\Gamma)\right)^{3}, \nu \cdot u=0, \operatorname{curl}_{\Gamma} u \in H^{-1 / 2}(\Gamma)\right),
\end{aligned}
$$


respectively. Finally, we introduce the trace space of $X\left(D, \Gamma_{2}\right)$ on $\Gamma$ by

$$
Y(\Gamma):=\left\{h \in\left(H^{-1 / 2}(\Gamma)\right)^{3}: \exists u \in H_{0}\left(\operatorname{curl}, B_{R}\right), \nu \times\left. u\right|_{\Gamma_{2}} \in L_{t}^{2}\left(\Gamma_{2}\right) \text { and } h=\nu \times\left. u\right|_{\Gamma}\right\},
$$

where the ball $B_{R}$ contains $D$ and $H_{0}\left(\operatorname{curl}, B_{R}\right)$ is the space of functions $u$ in $H\left(\operatorname{curl}, B_{R}\right)$ satisfying $\nu \times\left. u\right|_{S_{R}}=0$. As shown in $[4] Y(\Gamma)$ is a Banach space with respect to the norm

$$
\|h\|_{Y(\Gamma)}^{2}:=\inf \left\{\|u\|_{H\left(\operatorname{curl}, B_{R}\right)}^{2}+\|\nu \times u\|_{L_{t}^{2}\left(\Gamma_{2}\right)}^{2}\right\}
$$

where the infimum is taken over all functions $u \in H_{0}\left(\operatorname{curl}, B_{R}\right)$ such that $\nu \times\left. u\right|_{\Gamma_{2}} \in L_{t}^{2}\left(\Gamma_{2}\right)$ and $h=\nu \times\left. u\right|_{\Gamma} . Y(\Gamma)$ is a Hilbert space and coincides with $H_{\operatorname{div}}^{-1 / 2}(\Gamma) \cap L_{t}^{2}\left(\Gamma_{2}\right)$.

The direct scattering problem is a particular case of the following general transmission problem. Given $f \in Y(\Gamma), h \in Y(\Gamma), h_{1}=\left.h\right|_{\Gamma_{1}}, h_{2}=\left.h\right|_{\Gamma_{2}}$ find $E^{\mathrm{s}} \in X_{\mathrm{loc}}\left(D_{\mathrm{e}}, \Gamma_{2}\right)$, $E^{\text {int }} \in X\left(D, \Gamma_{2}\right), H^{\mathrm{s}} \in H_{\mathrm{loc}}\left(\operatorname{curl}, D_{\mathrm{e}}\right)$ and $H^{\text {int }} \in H(\operatorname{curl}, D)$ such that

$$
\begin{aligned}
\nabla \times E^{\mathrm{s}}-\mathrm{i} k H^{\mathrm{s}} & =0 \quad \text { and } \nabla \times H^{\mathrm{s}}+\mathrm{i} k E^{\mathrm{s}}=0 \quad \text { in } D_{\mathrm{e}}, \\
\nabla \times E^{\mathrm{int}}-\mathrm{i} k H^{\text {int }} & =0 \quad \text { and } \nabla \times H^{\mathrm{int}}+\mathrm{i} k n(x) E^{\mathrm{int}}=0 \quad \text { in } D, \\
\nu \times E^{\mathrm{s}}-\nu \times E^{\text {int }} & =f \quad \text { on } \Gamma, \\
\nu \times H^{\mathrm{s}}-\nu \times H^{\text {int }} & =h_{1} \quad \text { on } \Gamma_{1}, \\
\nu \times H^{\mathrm{s}}-\nu \times H^{\text {int }} & =\eta(x) E_{\mathrm{T}}^{\mathrm{s}}+h_{2} \quad \text { on } \Gamma_{2}, \\
\lim _{r \rightarrow \infty}\left(H^{\mathrm{s}} \times x-r E^{\mathrm{s}}\right) & =0,
\end{aligned}
$$

where by $u_{\mathrm{T}}$ we denote the tangential component of $u$ given by $u_{\mathrm{T}}=(\nu \times u) \times\left.\nu\right|_{\Gamma}$. We refer to (2.17) as problem (TP).

In order to arrive at a variational formulation of (TP) over a bounded domain, we introduce an artificial boundary $S_{R}$ that is the surface of the ball $B_{R}$ of radius $R$ such that the scatterer is contained in the interior of the ball. Furthermore, for given $f \in Y(\Gamma)$ we construct a function $E_{f} \in X\left(B_{R} \backslash \bar{D}, \Gamma_{2}\right)$ by solving the boundary-value problem

$$
\nabla \times \nabla \times E_{f}+E_{f}=0 \quad \text { in } B_{R} \backslash \bar{D}, \quad \nu \times\left. E_{f}\right|_{\Gamma}=f \quad \text { and } \quad \nu \times\left. E_{f}\right|_{S_{R}}=0 .
$$

Note that this problem is uniquely solvable in $H\left(\operatorname{curl}, B_{R} \cap D_{\mathrm{e}}\right)$ and by transforming this to a problem with homogeneous boundary data we see that

$$
\left\|E_{f}\right\|_{H\left(\operatorname{curl}, B_{R} \cap D_{\mathrm{e}}\right)} \leqslant C\|\tilde{E}\|_{H\left(\operatorname{curl}, B_{R} \cap D_{\mathrm{e}}\right)}
$$

for any function $\tilde{E} \in H_{0}\left(\operatorname{curl}, B_{R}\right)$ such that $\nu \times\left.\tilde{E}\right|_{\Gamma}=f$ and $\nu \times\left.\tilde{E}\right|_{S_{R}}=0$, where $C=$ $C\left(B_{R} \cap D_{\mathrm{e}}\right)$ is a positive constant. Now for all $\epsilon>0$, from the definition of $Y(\Gamma)$, we can choose a $\tilde{E}_{\epsilon}$ in $H_{0}\left(\operatorname{curl}, B_{R}\right)$ satisfying

$$
\left\|\tilde{E}_{\epsilon}\right\|_{H\left(\operatorname{curl}, B_{R}\right)}+\left\|\nu \times \tilde{E}_{\epsilon}\right\|_{L^{2}\left(\Gamma_{2}\right)} \leqslant\|f\|_{Y(\Gamma)}+\epsilon
$$

and since the above constant $C$ is independent of the choice of $\tilde{E}$ we obtain

$$
\left\|E_{f}\right\|_{X\left(B_{R} \backslash \bar{D}, \Gamma_{2}\right)} \leqslant C\|f\|_{Y(\Gamma)}, \quad \text { for some constant } C>0 \text {. }
$$


We now introduce the exterior Calderon operator $G_{\mathrm{e}}$ (cf. $\left.[\mathbf{1 5}, \mathbf{1 6}]\right)$, which is an isomorphism between $H_{\mathrm{div}}^{-1 / 2}\left(S_{R}\right)$ and $H_{\mathrm{div}}^{-1 / 2}\left(S_{R}\right)$ and maps a tangential vector field $\lambda$ on $S_{R}$ to $\hat{x} \times H^{\mathrm{s}}$ where $\left(E^{\mathrm{s}}, H^{\mathrm{s}}\right)$ satisfies

$$
\left.\begin{array}{rl}
\nabla \times E^{\mathrm{s}}-\mathrm{i} k H^{\mathrm{s}}=0 & \text { in } \mathbb{R}^{3} \backslash \bar{B}_{R}, \\
\nabla \times H^{\mathrm{s}}+\mathrm{i} k E^{\mathrm{s}}=0 & \text { in } \mathbb{R}^{3} \backslash \bar{B}_{R}, \\
\hat{x} \times E^{\mathrm{s}}=\lambda & \text { on } S_{R}, \\
\mathrm{~m}_{\infty}\left(H^{\mathrm{s}} \times x-r E^{\mathrm{s}}\right)=0 . &
\end{array}\right\}
$$

By expressing the magnetic fields in (TP) in terms of the electric fields, using the transmission conditions, the definition of $G_{\mathrm{e}}$ and integrating by parts, we obtain the following variational formulation for the electric field of (TP). Given $h \in Y(\Gamma)$, find $U \in X\left(B_{R}, \Gamma_{2}\right)$ satisfying

$$
\begin{gathered}
\int_{D}\left[(\nabla \times U) \cdot(\nabla \times \phi)-k^{2} n U \cdot \phi\right] \mathrm{d} v \\
+\int_{B_{R} \backslash \bar{D}}\left[(\nabla \times U) \cdot(\nabla \times \phi)-k^{2} U \cdot \phi\right] \mathrm{d} v \\
-\mathrm{i} k \int_{\Gamma_{2}} \eta U_{\mathrm{T}} \cdot \phi_{\mathrm{T}} \mathrm{d} s+\mathrm{i} k \int_{S_{R}} G_{\mathrm{e}}(\hat{x} \times U) \cdot \phi_{\mathrm{T}} \mathrm{d} s \\
=\int_{\Gamma} h \cdot \phi_{\mathrm{T}} \mathrm{d} s+\mathrm{i} k \int_{\Gamma_{2}} \eta E_{f T} \cdot \phi_{\mathrm{T}} \mathrm{d} s \\
\quad-\int_{B_{R} \backslash \bar{D}}\left[\left(\nabla \times E_{f}\right) \cdot(\nabla \times \phi)+E_{f} \cdot \phi\right] \mathrm{d} v \\
-\mathrm{i} k \int_{S_{R}} G_{\mathrm{e}}\left(\hat{x} \times E_{f}\right) \cdot \phi_{\mathrm{T}} \mathrm{d} s
\end{gathered}
$$

for every test function $\phi \in X\left(B_{R}, \Gamma_{2}\right)$. If $U$ is a solution of (2.21), then it is easy to show by choosing sufficiently smooth test functions that $E^{\text {int }}:=\left.U\right|_{D}$ and $E^{\mathrm{s}}:=\left.U\right|_{B_{R} \backslash \bar{D}}+E_{f}$ satisfy the differential equations for the electric fields of (TP) in $D$ and $B_{R} \backslash \bar{D}$, respectively, the transmission conditions on $\Gamma$ (with $H^{\mathrm{s}}=(1 / \mathrm{i} k) \nabla \times E^{\mathrm{s}}$ and $\left.H^{\text {int }}=(1 / \mathrm{i} k) \nabla \times E^{\text {int }}\right)$ and $\hat{x} \times\left(\nabla \times E^{\mathrm{s}}\right)=\mathrm{i} k G_{\mathrm{e}}\left(\hat{x} \times E^{\mathrm{s}}\right)$ on $S_{R}$.

A solution of the variational problem (2.21) (from now on we refer to it as (VTP)) and the corresponding magnetic fields $H^{\mathrm{s}}=(1 / \mathrm{i} k) \nabla \times E^{\mathrm{s}}, H^{\mathrm{int}}=(1 / \mathrm{i} k) \nabla \times E^{\text {int }}$ can be extended to a solution of (TP). Indeed, at the interface $S_{R}$ there is no jump of $\hat{x} \times E^{\mathrm{s}}$ and the link between $\hat{x} \times E^{\mathrm{s}}$ and $\hat{x} \times H^{\mathrm{s}}$ through the operator $G_{\mathrm{e}}$ shows that $\hat{x} \times H^{\mathrm{s}}$ has no jump either.

Theorem 2.1. The problems (TP) and (VTP) have at most one solution.

Proof. It suffices to show uniqueness for the problem (TP). Let $E^{\text {int }} \in X\left(D, \Gamma_{2}\right)$, $E^{\mathrm{s}} \in X_{\text {loc }}\left(D_{\mathrm{e}}, \Gamma_{2}\right)$ and $H^{\mathrm{int}}=(1 / \mathrm{i} k) \nabla \times E^{\mathrm{int}}, H^{\mathrm{s}}=(1 / \mathrm{i} k) \nabla \times E^{\mathrm{s}}$ be the solution of (TP) with boundary data $f \equiv 0$ and $h \equiv 0$. Taking the dot product of the equations of 
(TP) for the electric fields by $\bar{E}^{\text {int }}$ and $\bar{E}^{\text {s }}$, and then using integration by parts and the transmission conditions on $\Gamma$, we obtain

$$
\begin{aligned}
\mathrm{i} k \int_{S_{R}}\left(\nu \times \bar{E}^{\mathrm{s}}\right) \cdot H^{\mathrm{s}} \mathrm{d} s= & -\int_{S_{R}}\left[\nu \times\left(\nabla \times E^{\mathrm{s}}\right)\right] \cdot \overline{E^{\mathrm{s}} \mathrm{T}} \mathrm{d} s \\
= & \int_{B_{R} \backslash D}\left(\left|\nabla \times E^{\mathrm{s}}\right|^{2}-k^{2}\left|E^{\mathrm{s}}\right|^{2}\right) \mathrm{d} v \\
& \quad+\int_{D}\left(\left|\nabla \times E^{\mathrm{int}}\right|^{2}-k^{2} n\left|E^{\mathrm{int}}\right|^{2}\right) \mathrm{d} v-\mathrm{i} k \int_{\Gamma_{2}} \eta\left|E_{\mathrm{T}}^{\mathrm{s}}\right|^{2} \mathrm{~d} s
\end{aligned}
$$

After taking the imaginary part of $(2.22)$ and using the fact that $\operatorname{Im}(n)>0$ and $\eta>0$, we now obtain

$$
\operatorname{Re}\left(\int_{S_{R}}\left(\nu \times \bar{E}^{\mathrm{s}}\right) \cdot H^{\mathrm{s}} \mathrm{d} s\right)=-k \int_{D} \operatorname{Im}(n)\left|E^{\mathrm{int}}\right|^{2}-\frac{1}{k} \int_{\Gamma_{2}} \eta\left|E_{\mathrm{T}}^{\mathrm{s}}\right|^{2} \mathrm{~d} s \leqslant 0 .
$$

Hence from Theorem 6.10 of $[\mathbf{6}], E^{\mathrm{s}} \equiv 0$ and $H^{\mathrm{s}} \equiv 0$ in $D_{\mathrm{e}}$ and so the transmission conditions become simply the continuity of the tangential component of the electric and magnetic fields. Now we employ the unique continuation principle (cf. [18]) to deduce that $E^{\text {int }}$ and $H^{\text {int }}$ are both zero in $D$.

Now we prove the existence of a solution to (VTP). To this end we define the sesquilinear form $a: X\left(B_{R}, \Gamma_{2}\right) \times X\left(B_{R}, \Gamma_{2}\right) \rightarrow \mathbb{C}$ by

$$
\begin{aligned}
a(U, \phi):=(\nabla \times U, \nabla \times \phi)_{L^{2}\left(B_{R}\right)}- & k^{2}(\tilde{n} U, \phi)_{L^{2}\left(B_{R}\right)} \\
& +\mathrm{i} k\left\langle G_{\mathrm{e}}(\hat{x} \times U), \phi_{\mathrm{T}}\right\rangle_{L^{2}\left(S_{R}\right)}-\mathrm{i} k\left\langle\eta U_{\mathrm{T}}, \phi_{\mathrm{T}}\right\rangle_{L^{2}\left(\Gamma_{2}\right)},
\end{aligned}
$$

where $\tilde{n}:=n$ in $D$ and $\tilde{n}=1$ in $B_{R} \backslash \bar{D}$ and denote by $b(\phi)$ the right-hand side of (2.21) for $\phi \in X\left(B_{R}, \Gamma_{2}\right)$. Using the Schwarz inequality, the trace theorem and (2.19) it is easy to see that

$$
|b(\phi)| \leqslant C\left(\|h\|_{Y(\Gamma)}+\|f\|_{Y(\Gamma)}\right)\|\phi\|_{X\left(B_{R}, \Gamma_{2}\right)}, \quad \forall \phi \in X\left(B_{R}, \Gamma_{2}\right) .
$$

The existence of a solution to (VTP) is based on the technique used by Kirsch and Monk in $[\mathbf{1 5}, \mathbf{1 6}]$ for the case when $\eta \equiv 0$ (in this case our space $X\left(B_{R}, \Gamma_{2}\right)$ becomes simply $\left.H\left(\operatorname{curl}, B_{R}\right)\right)$. We follow their analysis, emphasizing the modifications due to the additional term on $\Gamma_{2}$. We first observe that any function $U \in X\left(B_{R}, \Gamma_{2}\right)$ such that $\nabla \times U=0$ in $B_{R}$ and $\nu \times\left. U\right|_{\Gamma_{2}}=0$ satisfies $U=\nabla p$ with $p \in S$, where $S:=\left\{p \in H^{1}\left(B_{R}\right):\left.p\right|_{\Gamma_{2}}=c\right\}$ and $c$ is a constant. (Note that the constants are possibly different on each disconnected component of $\Gamma_{2}$ and we can choose the value of $c$ on one component to vanish (see [4] or [16, Theorem 4.3] for details).)

The problem of the unique determination of $p \in S$ such that

$$
a(\nabla p, \nabla q):=-k^{2}(\tilde{n} \nabla p, \nabla q)_{L^{2}\left(B_{R}\right)}+\mathrm{i} k\left\langle G_{\mathrm{e}}(\hat{x} \times \nabla p), \nabla_{S_{R}} q\right\rangle_{L^{2}\left(S_{R}\right)}=b(\nabla q) \quad \forall q \in S
$$


now becomes exactly the one treated in Theorem 10.2 in [16] (see also [15]). In particular, there exist a unique $p \in S$ that solves (2.24) and satisfies

$$
\|\nabla p\|_{L^{2}\left(B_{R} \cap D_{\mathrm{e}}\right)} \leqslant C\|b\| .
$$

Now we can factor out $\nabla S$ from $X\left(B_{R} \cap D_{\mathrm{e}}, \Gamma_{2}\right)$. To this end, we define

$$
X_{0}:=\left\{U \in X\left(B_{R}, \Gamma_{2}\right):-k^{2}(\tilde{n} U, \nabla q)+\mathrm{i} k\left\langle G_{\mathrm{e}}(\hat{x} \times U), \nabla_{S_{R}} q\right\rangle=0 \forall q \in S\right\}
$$

and note that the condition in $X_{0}$ is a weak form of

$$
\left.\begin{array}{c}
\nabla \cdot(\tilde{n} U)=0 \quad \text { in } B_{R}, \\
k^{2} \hat{x} \cdot U=\mathrm{i} k \nabla_{S_{R}} \cdot G_{\mathrm{e}}(\hat{x} \times U) \quad \text { on } S_{R} .
\end{array}\right\}
$$

We then have that the space $X\left(B_{R} \cap D_{\mathrm{e}}, \Gamma_{2}\right)$ is the direct sum of $X_{0}$ and $\nabla S$, i.e.

$$
X\left(B_{R} \cap D_{\mathrm{e}}, \Gamma_{2}\right)=X_{0} \oplus \nabla S
$$

and furthermore that the projections onto the subspaces are bounded (the proof follows Lemma 10.3 in [16] or Lemma 0.1 in the Corrigendum to [15]).

We are now ready to analyse (VTP). First, by using expansions in spherical harmonics, one can show $\left[\mathbf{1 5}, \mathbf{1 6}\right.$, Lemma 10.5] that the exterior Calderon operator $G_{\mathrm{e}}$ can be split into two parts, $G_{\mathrm{e}} \lambda=G_{\mathrm{e}}^{1} \lambda+G_{\mathrm{e}}^{2} \lambda$ for $\lambda \in H_{\mathrm{div}}^{1 / 2}\left(S_{R}\right)$, such that the mapping

$$
\text { (a) } u \rightarrow G_{\mathrm{e}}^{1}(\hat{x} \times u) \text { is compact from } X_{0} \text { into } H_{\operatorname{div}}^{1 / 2}\left(S_{R}\right) \text {, }
$$

(b) $\quad \mathrm{i} k\left\langle G_{\mathrm{e}}^{2}(\hat{x} \times \lambda), \lambda_{\mathrm{T}}\right\rangle \geqslant 0$.

We now decompose $U=W+\nabla p$, where $W \in X_{0}$ and $\nabla p \in \nabla S$ and observe that $a(W, \nabla q)=0 \forall q \in S$ by the definition of $X_{0}$. Hence we can write

$$
a(\nabla p, \nabla q)+a(\nabla p, \psi)+a(W, \psi)=b(\nabla q)+b(\psi) \quad \forall \psi \in X_{0}, q \in S .
$$

After determining $p \in S$ from (2.24) it remains to find $W \in X_{0}$ by solving the equation

$$
a(W, \psi)=b(\psi)-a(\nabla p, \psi) \quad \forall \psi \in X_{0} .
$$

To this end we split the sesquilinear form $a(W, \psi)=a_{1}(W, \psi)+a_{2}(W, \psi)$, where

$$
\left.\begin{array}{c}
a_{1}(W, \psi):=(\nabla \times W, \nabla \times \psi)_{L^{2}\left(B_{R}\right)}+(U, \psi)_{L^{2}\left(B_{R}\right)} \\
-\mathrm{i} k\left\langle\eta U_{\mathrm{T}}, \psi_{\mathrm{T}}\right\rangle_{L^{2}\left(\Gamma_{2}\right)}+\mathrm{i} k\left\langle G_{\mathrm{e}}^{2}(\hat{x} \times U), \psi_{\mathrm{T}}\right\rangle_{L^{2}\left(\partial B_{R}\right)}, \\
a_{2}(W, \psi):=\mathrm{i} k\left\langle G_{\mathrm{e}}^{1}(\hat{x} \times U), \psi_{\mathrm{T}}\right\rangle_{L^{2}\left(S_{R}\right)}-\left(\left(k^{2} \tilde{n}+1\right) U, \psi\right)_{L^{2}\left(B_{R}\right)} .
\end{array}\right\}
$$

From the Cauchy-Schwarz inequality we have that

$$
\left|a_{1}(W, \psi)\right| \leqslant c_{1}\|W\|_{X}\|\psi\|_{X}, \quad c_{1}>0,
$$

and by taking the real and imaginary parts, using (2.28) and the fact that $\eta>0$ we have

$$
\left|a_{1}(W, \bar{W})\right| \geqslant c_{2}\|W\|_{X}^{2}, \quad c_{2}>0 .
$$

In order to treat the sesquilinear form $a_{2}(W, \psi)$ we need the following lemma. 
Lemma 2.2. The space $X_{0}$ is compactly imbedded in $L^{2}\left(B_{R}\right)$.

Proof. Consider a bounded sequence $\left\{U_{j}\right\}_{j=1}^{\infty}$ in $X_{0}$. Each function in $U_{j} \in X_{0}$ can be extended to all $\mathbb{R}^{3}$ by solving the exterior Maxwell problem

$$
\begin{aligned}
\nabla \times\left(\nabla \times V_{j}\right)-k^{2} V_{j} & =0 & & \text { in } \mathbb{R}^{3} \backslash \bar{B}_{R}, \\
\hat{x} \times V_{j} & =\hat{x} \times U_{j} & & \text { on } S_{R},
\end{aligned}
$$

together with the Silver-Müller radiation condition at infinity. The extended function $U_{j}^{\mathrm{e}}$ defined by

$$
U_{j}^{\mathrm{e}}= \begin{cases}U_{j} & \text { on } B_{R}, \\ V_{j} & \text { on } \mathbb{R}^{3} \backslash \bar{B}_{R}\end{cases}
$$

is in $H_{\text {loc }}\left(\right.$ curl, $\left.\mathbb{R}^{3}\right)$ since the tangential components are continuous across $S_{R}$. Due to the condition (2.26), the extended function has a well-defined divergence and

$$
\nabla \cdot\left(\tilde{n} U_{j}^{\mathrm{e}}\right)=0 \quad \text { in } \mathbb{R}^{3} .
$$

Now we choose a cut-off function $\chi \in C_{0}^{\infty}\left(\mathbb{R}^{3}\right)$ such that $\chi=1$ in $\bar{B}_{R}$ and is supported in $\Omega \supset \bar{B}_{R}$. From a result of Costabel [9] we have that the space

$$
H_{0}(\operatorname{curl}, \operatorname{div}, \Omega):=\left\{U \in H(\operatorname{curl}, \operatorname{div}, \Omega): \nu \times\left. U\right|_{\Gamma_{2}} \in L^{2}\left(\Gamma_{2}\right) \text { and } \nu \times\left. U\right|_{\partial \Omega}=0\right\},
$$

where $H(\operatorname{curl}, \operatorname{div}, \Omega)$ is the space of function $U \in L^{2}(\Omega)$ such that $\operatorname{curl} U \in L^{2}(\Omega)$ and $\operatorname{div} U \in L^{2}(\Omega)$, is continuously imbedded in $H^{1 / 2-\epsilon}(\Omega)$ for every $\epsilon>0$ and therefore the space

$$
\tilde{X}_{0}^{1}:=\left\{U \in X\left(\Omega, \Gamma_{2}\right): \nu \times\left. U\right|_{\partial \Omega}=0 \text { and }(U, \nabla \xi)_{L^{2}(\Omega)}=0, \forall \xi \in S\right\}
$$

is compactly imbedded in $\left(L^{2}(\Omega)\right)^{3}$. By using the technique of Theorem 4.7 in [16] or Proposition 2.28 in [5] we can extend this result to the space

$$
\tilde{X}_{0}^{\tilde{n}}:=\left\{U \in X\left(\Omega, \Gamma_{2}\right): \nu \times\left. U\right|_{\partial \Omega}=0 \text { and }(\tilde{n} U, \nabla \xi)_{L^{2}(\Omega)}=0, \forall \xi \in S\right\},
$$

and the result now follows from the fact that $\chi U_{j}^{\mathrm{e}} \in \tilde{X}_{0}^{\tilde{n}}$.

We are now ready to conclude the proof of the existence of a solution to (VTP). By an application of the Lax-Milgram lemma, $a_{1}$ gives rise to a bijective operator and by the compact imbedding of $X_{0}$ in $L^{2}\left(B_{R}\right)$ and (2.27) the second part $a_{2}$ gives rise to a compact operator. Then a standard argument implies that the Fredholm alternative is applicable, which together with the uniqueness Theorem 2.1 shows that there exists a unique solution $U$ of $(\mathrm{VTP})$. Hence $E^{\mathrm{int}}=\left.U\right|_{D}, H^{\mathrm{int}}=(1 / \mathrm{i} k) \nabla \times E^{\mathrm{int}}$ and the unique extension $E^{\mathrm{s}}$ to $D_{\mathrm{e}}$ of $\left.U\right|_{B_{R} \backslash \bar{D}}+E_{f}$ and $H^{\mathrm{s}}=(1 / \mathrm{i} k) \nabla \times E^{\mathrm{s}}$ are the unique solution of (TP). So we have proved the following result provided $D, n$ and $\eta$ satisfy the assumptions of $\S 2.1$. 
Theorem 2.3. The transmission problem (TP) has a unique solution $E^{\mathrm{int}} \in X(D, \Gamma)$, $E^{\mathrm{s}} \in X\left(D_{\mathrm{e}}, \Gamma\right), H^{\mathrm{int}} \in H(\operatorname{curl}, D), H^{\mathrm{s}} \in H\left(\operatorname{curl}, D_{\mathrm{e}}\right)$. Moreover, it satisfies

$$
\begin{aligned}
\left\|E^{\mathrm{int}}\right\|_{X(D, \Gamma)}+\left\|E^{\mathrm{s}}\right\|_{X\left(B_{R} \backslash \bar{D}, \Gamma\right)}+\left\|H^{\mathrm{int}}\right\|_{H(\operatorname{curl}, D)}+\left\|H^{\mathrm{s}}\right\|_{H\left(\operatorname{curl}, B_{R} \backslash \bar{D}\right)} \\
\leqslant C\left(\|f\|_{Y(\Gamma)}+\|h\|_{Y(\Gamma)}\right),
\end{aligned}
$$

for some positive constant $C$ depending on $R$ but not on $f$ and $h$.

\subsection{Regularity of the solution of the direct problem}

Let $\mathcal{K}=\nabla \nu$ and $\mathcal{H}=\frac{1}{2} \nabla \cdot \nu$ on $\Gamma$ denote the curvature and mean curvature, respectively, which are bounded for a $C^{2}$-smooth boundary, and let $H_{t}^{1 / 2}(\Gamma)$ be the space of the tangential fields in $H^{1 / 2}(\Gamma)$. In order to prove our desired uniqueness theorem in $\S 4$, we need to establish a regularity result for the solution of (TP).

We begin with the following technical lemma [17, Lemma 5.4.2].

Lemma 2.4. Let $\Omega$ be a bounded domain having a $C^{2}$-smooth boundary $\Gamma$ and let $\nu$ be the outward normal vector. Then for any two vectors $U$ and $V$ in $\left(H^{1}(\Omega)\right)^{3}$ we have

$$
\begin{aligned}
\int_{\Omega}[(\nabla U & \cdot \nabla V)-(\nabla \times U) \cdot(\nabla \times V)-(\nabla \cdot U)(\nabla \cdot V)] \mathrm{d} v \\
& =-\int_{\Gamma}\left[\nabla_{\Gamma} \cdot U_{\mathrm{T}}(V \cdot \nu)+\nabla_{\Gamma} \cdot V_{\mathrm{T}}(U \cdot \nu)+2 \mathcal{H}(U \cdot \nu)(V \cdot \nu)+\left(\mathcal{K} U_{\mathrm{T}} \cdot V_{\mathrm{T}}\right)\right] \mathrm{d} s .
\end{aligned}
$$

We are now ready to prove a regularity result for the solution of the transmission problem (TP) under some restrictions on the behaviour of the surface conductivity $\eta$ at the boundary $\Pi$ of $\Gamma_{2}$ on $\Gamma$.

Theorem 2.5. Suppose that $\nabla_{\Gamma} \cdot f \in H^{1 / 2}(\Gamma), h \in H_{t}^{1 / 2}(\Gamma)$, and $\eta$ and $\nabla_{\Gamma} \eta$ act as multipliers in $H^{-1 / 2}(\Gamma)$ and $H_{t}^{-1 / 2}(\Gamma)$, respectively. Then the magnetic fields of the solution to (TP) satisfy $H^{\text {int }} \in\left(H^{1}(D)\right)^{3}$ and $H^{\mathrm{s}} \in\left(H_{\mathrm{loc}}^{1}\left(D_{\mathrm{e}}\right)\right)^{3}$.

Proof. The magnetic fields $H^{\mathrm{s}} \in H\left(\operatorname{curl}, D_{\mathrm{e}}\right)$ and $H^{\mathrm{int}} \in H(\operatorname{curl}, D)$ of the solution to the (TP) satisfy

$$
\begin{aligned}
& \nabla \times\left(\nabla \times H^{\mathrm{s}}\right)-k^{2} H^{\mathrm{s}}=0, \quad \nabla \cdot H^{\mathrm{s}}=0 \quad \text { in } D_{\mathrm{e}}, \\
& \nabla \times\left(n^{-1} \nabla \times H^{\mathrm{int}}\right)-k^{2} H^{\mathrm{int}}=0, \quad \nabla \cdot H^{\mathrm{int}}=0 \quad \text { in } D
\end{aligned}
$$

and the transmission conditions

$$
\left.\begin{array}{rlrl}
\nu \cdot H^{\mathrm{s}}-\nu \cdot H^{\mathrm{int}} & =\frac{\mathrm{i}}{k} \nabla_{\Gamma} \cdot f & \text { on } \Gamma, & \\
H_{\mathrm{T}}^{\mathrm{s}}-H_{\mathrm{T}}^{\mathrm{int}} & =h_{1} \times \nu & \text { on } \Gamma_{1}, & \\
H_{\mathrm{T}}^{\mathrm{s}}-H_{\mathrm{T}}^{\mathrm{int}} & =-\frac{\mathrm{i}}{k} \eta(x)\left[\nu \times\left(\nabla \times H^{\mathrm{s}}\right)\right]+h_{2} \times \nu & \text { on } \Gamma_{2},
\end{array}\right\}
$$


which are obtained by first applying the surface divergence to $(2.17 c)$ and then using $\nabla_{\Gamma} \cdot\left(\nu \times E^{\mathrm{s}}\right)=-\nu \cdot\left(\nabla \times E^{\mathrm{s}}\right)=-\mathrm{i} k\left(\nu \cdot H^{\mathrm{s}}\right)$.

Now we apply Lemma 2.4 for smooth $H^{\text {int }}, \bar{H}^{\text {int }}$ and $H^{\mathrm{s}}, \bar{H}^{\mathrm{s}}$ in $D$ and $B_{R} \backslash \bar{D}$, respectively, to estimate the $L^{2}$ norm of the gradient. The desired result is then obtained by employing a denseness argument. In particular, we have

$$
\begin{aligned}
\int_{D}\left|\nabla H^{\mathrm{int}}\right|^{2} \mathrm{~d} v+\int_{B_{R} \backslash \bar{D}}\left|\nabla H^{\mathrm{s}}\right|^{2} \mathrm{~d} v & \\
=\int_{D}\left|\nabla \times H^{\mathrm{int}}\right|^{2} \mathrm{~d} v+\int_{B_{R} \backslash \bar{D}}\left|\nabla \times H^{\mathrm{s}}\right|^{2} \mathrm{~d} v & -\int_{\Gamma}\left[\nabla_{\Gamma} \cdot H_{\mathrm{T}}^{\mathrm{int}}\left(\bar{H}^{\mathrm{int}} \cdot \nu\right)+\nabla_{\Gamma} \cdot \bar{H}_{\mathrm{T}}^{\mathrm{int}}\left(H^{\mathrm{int}} \cdot \nu\right)\right. \\
& \left.+2 \mathcal{H}\left(H^{\mathrm{int}} \cdot \nu\right)\left(\bar{H}^{\mathrm{int}} \cdot \nu\right)+\left(\mathcal{K} H_{\mathrm{T}}^{\mathrm{int}} \cdot \bar{H}_{\mathrm{T}}^{\mathrm{int}}\right)\right] \mathrm{d} s \\
& +\int_{\Gamma}\left[\nabla_{\Gamma} \cdot H_{\mathrm{T}}^{\mathrm{s}}\left(\overline{H^{\mathrm{s}}} \cdot \nu\right)+\nabla_{\Gamma} \cdot \overline{H_{\mathrm{s}}^{\mathrm{s}}}\left(H^{\mathrm{s}} \cdot \nu\right)\right. \\
& \left.+\mathcal{H}\left(H^{\mathrm{s}} \cdot \nu\right)\left(\bar{H}^{\mathrm{s}} \cdot \nu\right)+\left(\mathcal{K} H_{\mathrm{T}}^{\mathrm{s}} \cdot \bar{H}_{\mathrm{T}}^{\mathrm{s}}\right)\right] \mathrm{d} s \\
& -\int_{S_{R}}\left[\nabla_{S_{R}} \cdot H_{\mathrm{T}}^{\mathrm{s}}\left(\overline{H^{\mathrm{s}}} \cdot \hat{x}\right)+\nabla_{S_{R}} \cdot \bar{H}_{\mathrm{s}}\left(H^{\mathrm{s}} \cdot \hat{x}\right)\right. \\
& \left.+2 \mathcal{H}\left(H^{\mathrm{s}} \cdot \hat{x}\right)\left(\bar{H}^{\mathrm{s}} \cdot \hat{x}\right)+\left(\mathcal{K} H_{\mathrm{T}}^{\mathrm{s}} \cdot \bar{H}_{\mathrm{T}}^{\mathrm{s}}\right)\right] \mathrm{d} \hat{x} .
\end{aligned}
$$

By using the Schwartz inequality and the fact that $\mathcal{K}$, and $\mathcal{H}$ are bounded we obtain

$$
\begin{aligned}
\int_{D} \mid \nabla & \left.H^{\mathrm{int}}\right|^{2} \mathrm{~d} v+\int_{B_{R} \backslash \bar{D}}\left|\nabla H^{\mathrm{s}}\right|^{2} \mathrm{~d} v \\
\leqslant & \nabla \times H^{\mathrm{int}}\left\|_{L^{2}(D)}^{2}+\right\| \nabla \times H^{\mathrm{s}} \|_{L^{2}\left(B_{R} \backslash \bar{D}\right)}^{2} \\
& +c\left\|H^{\mathrm{int}} \cdot \nu\right\|_{H^{-1 / 2}(\Gamma)}\left\|H^{\mathrm{int}} \cdot \nu\right\|_{H^{1 / 2}(\Gamma)}+c\left\|H^{\mathrm{s}} \cdot \nu\right\|_{H^{-1 / 2}(\Gamma)}\left\|H^{\mathrm{s}} \cdot \nu\right\|_{H^{1 / 2}(\Gamma)} \\
& +c\left\|H^{\mathrm{s}} \cdot \hat{x}\right\|_{H^{-1 / 2}\left(S_{R}\right)}\left\|H^{\mathrm{s}} \cdot \hat{x}\right\|_{H^{1 / 2}\left(S_{R}\right)}+c\left\|\nabla_{S_{R}} \cdot H_{\mathrm{T}}^{\mathrm{s}}\right\|_{H^{-1 / 2}\left(S_{R}\right)}\left\|H^{\mathrm{s}} \cdot \hat{x}\right\|_{H^{1 / 2}\left(S_{R}\right)} \\
& +2\left|\int_{\Gamma}\left[\nabla_{\Gamma} \cdot H_{\mathrm{T}}^{\mathrm{s}}\left(\overline{H^{\mathrm{s}}} \cdot \nu\right)-\nabla_{\Gamma} \cdot H_{\mathrm{T}}^{\mathrm{int}}\left(\bar{H}^{\mathrm{int}} \cdot \nu\right)\right] \mathrm{d} s\right|
\end{aligned}
$$

We now note that by expanding the magnetic field $H$ of the solution of (TP) in spherical harmonics $[\mathbf{6}, \mathbf{1 5}]$, we can see that $\left\|\nabla_{\Gamma} \cdot H_{\mathrm{T}}^{\mathrm{s}}\right\|_{H^{-1 / 2}\left(S_{R}\right)}$ is bounded. Next we use the transmission conditions (2.34) to see that

$$
\begin{array}{r}
\int_{\Gamma}\left[\nabla_{\Gamma} \cdot H_{\mathrm{T}}^{\mathrm{s}}\left(\overline{H^{\mathrm{s}}} \cdot \nu\right)-\nabla_{\Gamma} \cdot H_{\mathrm{T}}^{\mathrm{int}}\left(\bar{H}^{\mathrm{int}} \cdot \nu\right)\right] \mathrm{d} s \\
=-\frac{\mathrm{i}}{k} \int_{\Gamma} \nabla_{\Gamma} \cdot H_{\mathrm{T}}^{\mathrm{s}}\left(\nabla_{\Gamma} \cdot \bar{f}\right) \mathrm{d} s+\int_{\Gamma} \nabla_{\Gamma} \cdot(h \times \nu)\left(\bar{H}^{\mathrm{int}} \cdot \nu\right) \mathrm{d} s \\
\quad-\frac{\mathrm{i}}{k} \int_{\Gamma_{2}} \nabla_{\Gamma} \cdot\left[\eta\left(\nu \times \nabla \times H^{\mathrm{s}}\right)\right]\left(\bar{H}^{\mathrm{int}} \cdot \nu\right) \mathrm{d} s
\end{array}
$$


and hence

$$
\begin{gathered}
\left|\int_{\Gamma}\left[\nabla_{\Gamma} \cdot H_{\mathrm{T}}^{\mathrm{s}}(\bar{H} \cdot \nu)-\nabla_{\Gamma} \cdot H_{\mathrm{T}}^{\mathrm{int}}\left(\bar{H}^{\mathrm{int}} \cdot \nu\right)\right] \mathrm{d} s\right| \\
\leqslant c\left\|\left(\nabla_{\Gamma} \cdot H_{\mathrm{T}}^{\mathrm{s}}\right)\right\|_{H^{-1 / 2}(\Gamma)}\left\|\nabla_{\Gamma} \cdot f\right\|_{H^{1 / 2}(\Gamma)} \\
\quad+c\left\|\nabla_{\Gamma} \cdot(h \times \nu)\right\|_{H^{-1 / 2}(\Gamma)}\left\|H^{\mathrm{int}} \cdot \nu\right\|_{H^{1 / 2}(\Gamma)} \\
\quad+c\left\|\nabla_{\Gamma} \cdot\left[\eta\left(\nu \times \nabla \times H^{\mathrm{s}}\right)\right]\right\|_{H^{-1 / 2}(\Gamma)}\left\|H^{\mathrm{int}} \cdot \nu\right\|_{H^{1 / 2}(\Gamma)}
\end{gathered}
$$

Simple computations show that

$$
\nabla_{\Gamma} \cdot\left[\eta\left(\nu \times\left(\nabla \times H^{\mathrm{s}}\right)\right)\right]=\eta \nabla_{\Gamma} \cdot\left[\nu \times\left(\nabla \times H^{\mathrm{s}}\right)\right]+\nabla_{\Gamma} \eta \cdot\left[\nu \times\left(\nabla \times H^{\mathrm{s}}\right)\right] \quad \text { on } \Gamma_{2} .
$$

From (2.38) and the assumptions on $\eta$ and $\nabla_{\Gamma} \eta$ we have that for $H^{\mathrm{s}} \in H\left(\operatorname{curl}, B_{R} \backslash \bar{D}\right)$ both $\eta \nabla_{\Gamma} \cdot\left[\nu \times\left(\nabla \times H^{\mathrm{s}}\right)\right]$ and $\nabla_{\Gamma} \eta \cdot\left[\nu \times\left(\nabla \times H^{\mathrm{s}}\right)\right]$ are in $H^{-1 / 2}(\Gamma)$, whence $\| \nabla_{\Gamma} \cdot[\eta(\nu \times$ $\left.\left.\left(\nabla \times H^{\mathrm{s}}\right)\right)\right] \|_{H^{-1 / 2}}(\Gamma)$ is bounded. Now combining (2.35) and (2.37) we obtain that

$$
\begin{aligned}
& \int_{D}\left|\nabla H^{\mathrm{int}}\right|^{2} \mathrm{~d} v+\int_{B_{R} \backslash \bar{D}}\left|\nabla H^{\mathrm{s}}\right|^{2} \mathrm{~d} v \\
& \leqslant \| \nabla \times H^{\mathrm{int}}\left\|_{L^{2}(D)}+\right\| \nabla \times H^{\mathrm{s}} \|_{L^{2}\left(B_{R} \backslash \bar{D}\right)} \\
&+c\left\|H^{\mathrm{int}} \cdot \nu\right\|_{H^{-1 / 2}(\Gamma)}\left\|H^{\mathrm{int}} \cdot \nu\right\|_{H^{1 / 2}(\Gamma)}+c\left\|H^{\mathrm{s}} \cdot \nu\right\|_{H^{-1 / 2}(\Gamma)}\left\|H^{\mathrm{s}} \cdot \nu\right\|_{H^{1 / 2}(\Gamma)} \\
&+c\left\|H^{\mathrm{s}} \cdot \hat{x}\right\|_{H^{-1 / 2}\left(S_{R}\right)}\left\|H^{\mathrm{s}} \cdot \hat{x}\right\|_{H^{1 / 2}\left(S_{R}\right)}+c\left\|\nabla_{S_{R}} \cdot H_{\mathrm{T}}^{\mathrm{s}}\right\|_{H^{-1 / 2}\left(S_{R}\right)}\left\|H^{\mathrm{s}} \cdot \hat{x}\right\|_{H^{1 / 2}\left(S_{R}\right)} \\
&+c\left\|\nabla_{\Gamma} \cdot(h \times \nu)\right\|_{H^{-1 / 2}(\Gamma)}\left\|H^{\mathrm{int}} \cdot \nu\right\|_{H^{1 / 2}(\Gamma)} \\
&+c\left\|\left(\nabla_{\Gamma} \cdot H_{\mathrm{T}}^{\mathrm{s}}\right)\right\|_{H^{-1 / 2}(\Gamma)}\left\|\nabla_{\Gamma} \cdot f\right\|_{H^{1 / 2}(\Gamma)} \\
&+c\left\|\nabla_{\Gamma} \cdot\left[\eta\left(\nu \times \nabla \times H^{\mathrm{s}}\right)\right]\right\|_{H^{-1 / 2}(\Gamma)}\left\|H^{\mathrm{int}} \cdot \nu\right\|_{H^{1 / 2}(\Gamma)} .
\end{aligned}
$$

The first two terms of (2.39) are bounded by the $H(\operatorname{curl}, D)$ and $H\left(\operatorname{curl}, B_{R} \backslash D\right)$ norms, respectively. In order to estimate the third term, we use the inequality

$$
2\left\|H^{\mathrm{int}} \cdot \nu\right\|_{H^{1 / 2}(\Gamma)}\left\|H^{\mathrm{int}} \cdot \nu\right\|_{H^{-1 / 2}(\Gamma)} \leqslant \epsilon\left\|H^{\mathrm{int}} \cdot \nu\right\|_{H^{1 / 2}(\Gamma)}^{2}+\frac{1}{\epsilon}\left\|H^{\mathrm{int}} \cdot \nu\right\|_{H^{-1 / 2}(\Gamma)}^{2} .
$$

It follows from the trace theorem that when $\epsilon$ is small enough the first term on the right-hand side of (2.40) is dominated by $\left\|H^{\text {int }}\right\|_{H^{1}}$ and the second term is bounded with respect to the $H(\operatorname{curl}, D)$ norm. The same technique can be applied to each of the remaining terms in (2.39). Note that by assumption $\|h\|_{H^{1 / 2}(\Gamma)}$ and $\left\|\nabla_{\Gamma} \cdot f\right\|_{H^{1 / 2}(\Gamma)}$ are bounded. Hence the above analysis shows that $H^{\text {int }} \in\left(H^{1}(D)\right)^{3}$ and $H \in\left(H^{1}\left(B_{R} \backslash \bar{D}\right)\right)^{3}$ and

$$
\begin{aligned}
& \left\|H^{\mathrm{int}}\right\|_{H^{1}(D)}+\left\|H^{\mathrm{s}}\right\|_{H^{1}\left(B_{R} \backslash \bar{D}\right)} \\
& \quad \leqslant C\left(\left\|H^{\mathrm{int}}\right\|_{H(\operatorname{curl}, D)}+\left\|H^{\mathrm{s}}\right\|_{H\left(\operatorname{curl}, B_{R} \backslash \bar{D}\right)}+\|h\|_{H_{t}^{1 / 2}(\Gamma)}+\left\|\nabla_{\Gamma} \cdot f\right\|_{H^{1 / 2}(\Gamma)}\right),
\end{aligned}
$$

with $C$ a positive constant depending on $R$ but not on $H, f$ and $h$. Note that $\left\|\hat{x} \cdot H^{\mathrm{s}}\right\|_{H^{1 / 2}\left(S_{R}\right)}$ becomes arbitrarily small for arbitrarily large $R$ since $H^{\mathrm{s}}$ satisfies the radiation condition. 
The same type of regularity can be obtained for the electric fields $E^{\text {int }}, E^{\mathrm{s}}$ of the solution to (TP).

Theorem 2.6. Suppose that $f \in H_{t}^{1 / 2}(\Gamma), h \in H_{t}^{1 / 2}(\Gamma), \nabla_{\Gamma} \cdot h \in H^{1 / 2}(\Gamma)$, and $1 / \eta$ and $\nabla_{\Gamma}(1 / \eta)$ act as multipliers in $H^{-1 / 2}(\Gamma)$ and $H^{-1 / 2}(\Gamma)_{t}$, respectively. Then the electric fields of the solution to $(\mathrm{TP})$ satisfy $E^{\mathrm{int}} \in\left(H^{1}(D)\right)^{3}$ and $E^{\mathrm{s}} \in\left(H_{\mathrm{loc}}^{1}\left(D_{\mathrm{e}}\right)\right)$.

Proof. The proof uses the same techniques as in the previous theorem. The transmission conditions for $E^{\text {int }}, E^{\mathrm{s}}$ now become

$$
\left.\begin{array}{rl}
E_{\mathrm{T}}^{\mathrm{s}}-E_{\mathrm{T}}^{\mathrm{int}} & =f \times \nu \quad \text { on } \Gamma, \\
-\nu \cdot\left(n E^{\mathrm{int}}\right) & =-\frac{\mathrm{i}}{k} \nabla_{\Gamma} \cdot h_{1} \quad \text { on } \Gamma_{1}, \\
-\nu \cdot\left(n E^{\mathrm{int}}\right) & =-\frac{\mathrm{i}}{k} \nabla_{\Gamma} \cdot\left(\eta E_{\mathrm{T}}^{\mathrm{s}}+h_{2}\right) \quad \text { on } \Gamma_{2} .
\end{array}\right\}
$$

Avoiding the repetition of the same procedure, we note only that some slight modifications are needed due to the fact that $\nabla \cdot\left(n E^{\text {int }}\right)=0$ (cf. [17, Theorem 5.4.4] for the equation corresponding to (2.33) in this case). In addition we need to make use of the relation

$$
\nabla_{\Gamma} \cdot E_{\mathrm{T}}^{\mathrm{s}}=\frac{\mathrm{i}}{k} \nabla_{\Gamma} \cdot\left[\frac{1}{\eta}\left(\nu \times\left(\nabla \times E^{\mathrm{s}}\right)-\nu \times\left(\nabla \times E^{\mathrm{int}}\right)\right)\right]-\frac{\mathrm{i}}{k} \nabla_{\Gamma} \cdot\left(\frac{1}{\eta} h\right) \quad \text { on } \Gamma_{2}
$$

in order to estimate the integral over $\Gamma_{2}$, where the assumptions on $1 / \eta$ and $\nabla_{\Gamma}(1 / \eta)$ are used.

The corresponding norm estimate for the electric fields is

$$
\begin{aligned}
\left\|E^{\mathrm{int}}\right\|_{H^{1}(D)}+\left\|E^{\mathrm{s}}\right\|_{H^{1}\left(B_{R} \backslash \bar{D}\right)} \leqslant & C\left(\left\|E^{\mathrm{int}}\right\|_{H(\operatorname{curl}, D)}+\left\|E^{\mathrm{s}}\right\|_{H\left(\operatorname{curl}, B_{R} \backslash \bar{D}\right)}\right. \\
& \left.+\|f\|_{H_{t}^{1 / 2}(\Gamma)}+\left\|\nabla_{\Gamma} \cdot h\right\|_{H^{1 / 2}(\Gamma)}+\|h\|_{H_{t}^{1 / 2}(\Gamma)}\right) .
\end{aligned}
$$

We end this section by reformulating the above regularity result in a form which will be used later on (see also [17, Theorem 5.4.4]).

Remark 2.7. It is easy to see that the conclusions of Theorems 2.5 and 2.6 are also valid if we merely require that the fields satisfy

$$
\begin{aligned}
& H^{\mathrm{s}} \in\left(L_{\mathrm{loc}}^{2}\left(D_{\mathrm{e}}\right)\right)^{3}, \quad \nabla \times H^{\mathrm{s}} \in\left(L_{\mathrm{loc}}^{2}\left(D_{\mathrm{e}}\right)\right)^{3}, \quad \nabla \cdot H^{\mathrm{s}} \in L_{\mathrm{loc}}^{2}\left(D_{\mathrm{e}}\right), \\
& H^{\mathrm{int}} \in\left(L^{2}(D)\right)^{3}, \quad \nabla \times H^{\mathrm{int}} \in\left(L^{2}(D)\right)^{3}, \quad \nabla \cdot H^{\mathrm{int}} \in L^{2}(D)
\end{aligned}
$$

together with (2.34), and

$$
\begin{aligned}
& E^{\mathrm{s}} \in\left(L_{\mathrm{loc}}^{2}\left(D_{\mathrm{e}}\right)\right)^{3}, \quad \nabla \times E^{\mathrm{s}} \in\left(L_{\mathrm{loc}}^{2}\left(D_{\mathrm{e}}\right)\right)^{3}, \quad \nabla \cdot E^{\mathrm{s}} \in L_{\mathrm{loc}}^{2}\left(D_{\mathrm{e}}\right), \\
& E^{\text {int }} \in\left(L^{2}(D)\right)^{3}, \quad \nabla \times E^{\text {int }} \in\left(L^{2}(D)\right)^{3}, \quad \nabla \cdot\left(n E^{\text {int }}\right) \in L^{2}(D)
\end{aligned}
$$

together with (2.42), respectively. 


\section{The interior transmission problem}

Before proceeding with the uniqueness of the inverse problem we need to study the interior transmission problem (referred to as (ITP)) corresponding to the transmission problem of the previous section. (For the relation of the interior transmission problem with the inverse scattering problem, see $[\mathbf{6}, \mathbf{1 1}])$. Throughout this section we assume that $D$ is simply connected. This is not a restriction since as will be seen in the next section we need only to consider (ITP) in each connected component of $D$ separately. The interior transmission problem is given $D, n$ and $\eta$ as in the direct problem and given $f \in Y(\Gamma), h \in Y(\Gamma), h_{1}=\left.h\right|_{\Gamma_{1}}, h_{2}=\left.h\right|_{\Gamma_{2}}$ find $E, E^{\text {int }} \in X\left(D, \Gamma_{2}\right), H, H^{\text {int }} \in H(\operatorname{curl}, D)$ satisfying

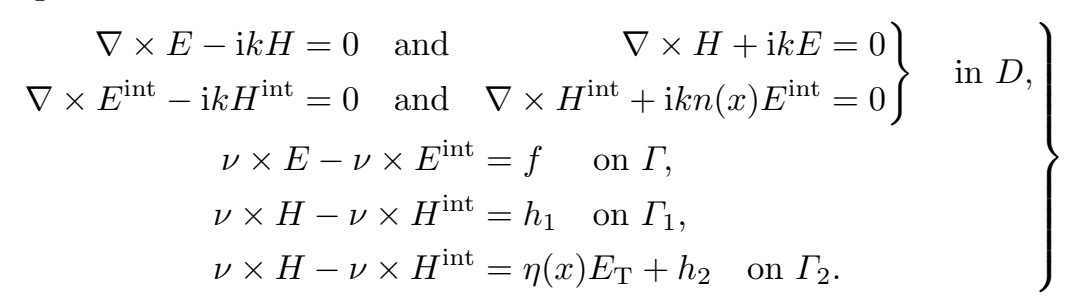

Our goal is to show that a slightly modified interior transmission problem has a unique solution that depends continuously on the data. It turns out that this modified interior transmission problem gives the necessary tool to prove the main result of this paper, i.e. the shape of a penetrable (possibly) partly coated anisotropic obstacle is uniquely determined by the far-field data.

\subsection{The modified interior transmission problem}

Let $m$ be a positive number, $F_{1}, F_{2} \in\left(L^{2}(D)\right)^{3}, f \in Y(\Gamma), h \in Y(\Gamma)$ and $h_{1}=\left.h\right|_{\Gamma_{1}}$, $h_{2}=\left.h\right|_{\Gamma_{2}}$. We want to find $H, H^{\text {int }} \in H(\operatorname{curl}, D)$ with $\nu \times\left.(\nabla \times H)\right|_{\Gamma_{2}} \in L_{t}^{2}\left(\Gamma_{2}\right)$ satisfying

$$
\begin{aligned}
& \left.\begin{array}{r}
\nabla \times(\nabla \times H)+H=F_{1} \\
\nabla \times n^{-1}\left(\nabla \times H^{\text {int }}\right)+m H^{\text {int }}=F_{2}
\end{array}\right\} \quad \text { in } D, \\
& \nu \times(\nabla \times H)-\nu \times n^{-1}\left(\nabla \times H^{\mathrm{int}}\right)=f \quad \text { on } \Gamma, \\
& H_{\mathrm{T}}-H_{\mathrm{T}}^{\mathrm{int}}=h_{1} \times \nu \quad \text { on } \Gamma_{1}, \\
& H_{\mathrm{T}}-H_{\mathrm{T}}^{\mathrm{int}}=-\frac{\mathrm{i}}{k} \eta(x)[\nu \times(\nabla \times H)]+h_{2} \times \nu \quad \text { on } \Gamma_{2} .
\end{aligned}
$$

We will reformulate (3.1) as a variational problem. To this end we introduce the sesquilinear form $\mathcal{A}$ defined on $H(\operatorname{curl}, D) \times X\left(D, \Gamma_{2}\right)$ by

$$
\begin{aligned}
\mathcal{A}(H, V ; \Phi, \Psi)=\int_{D} & {[(\nabla \times H) \cdot(\nabla \times \Phi)+H \cdot \Phi] \mathrm{d} x+\int_{\Gamma} H_{\mathrm{T}} \cdot(\nu \times \Psi) \mathrm{d} s } \\
& +\int_{D}\left[\frac{1}{m}(\nabla \times V) \cdot(\nabla \times \Psi)+n V \cdot \Psi\right] \mathrm{d} x \\
& +\int_{\Gamma}(\nu \times V) \cdot \Phi_{\mathrm{T}} \mathrm{d} s+\frac{\mathrm{i}}{k} \int_{\Gamma_{2}} \eta(\nu \times V) \cdot(\nu \times \Psi) \mathrm{d} s,
\end{aligned}
$$


where $(H, V)$ and $(\Phi, \Psi)$ are in $H(\operatorname{curl}, D) \times X\left(D, \Gamma_{2}\right)$. We also introduce for $(\Phi, \Psi) \in$ $H(\operatorname{curl}, D) \times X\left(D, \Gamma_{2}\right)$ the antilinear form

$$
\begin{aligned}
L(\Phi, \Psi)=\int_{D} & {\left[F_{1} \cdot \Phi+\frac{1}{m} F_{2} \cdot(\nabla \times \Psi)\right] \mathrm{d} x } \\
& +\int_{\Gamma}(h \times \nu) \cdot(\nu \times \Psi) \mathrm{d} s-\int_{\Gamma} f \cdot \Phi_{\mathrm{T}} \mathrm{d} s-\frac{\mathrm{i}}{k} \int_{\Gamma_{2}} \eta f \cdot(\nu \times \Psi) \mathrm{d} s .
\end{aligned}
$$

Note that the integrals over $\Gamma$ are interpreted as the duality between $H_{\mathrm{div}}^{-1 / 2}(\Gamma)$ and $H_{\text {curl }}^{-1 / 2}(\Gamma)$, while the integral over $\Gamma_{2}$ is the $L^{2}\left(\Gamma_{2}\right)$ scalar product (note that $h \times \nu$ is in $H_{\text {curl }}^{-1 / 2}(\Gamma)$ since $\left.h \in H_{\operatorname{div}}^{-1 / 2}(\Gamma)\right)$.

The variational formulation of problem (3.2) is find $(H, V) \in H(\operatorname{curl}, D) \times X\left(D, \Gamma_{2}\right)$ such that

$$
\mathcal{A}(H, V ; \Phi, \Psi)=L(\Phi, \Psi), \quad \forall(\Phi, \Psi) \in H(\operatorname{curl}, D) \times X\left(D, \Gamma_{2}\right) .
$$

The following theorem proves the equivalence between the existence of a solution to problems (3.2) and (3.5).

\section{Theorem 3.1.}

(a) If $\left(H, H^{\mathrm{int}}\right)$ is a solution to (3.2), then $(H, V)$ with $V:=n^{-1}\left(\nabla \times H^{\mathrm{int}}\right)$ is a solution to (3.5).

(b) Conversely, if $(H, V)$ is a solution to (3.5), then $\left(H, H^{\mathrm{int}}\right)$ with

$$
H^{\mathrm{int}}:=-\frac{1}{m}(\nabla \times V)+\frac{F_{2}}{m}
$$

is a solution to (3.2).

Proof. (a) Let $\left(H, H^{\text {int }}\right)$ be a solution of problem (3.2) and set $V:=n^{-1} \nabla \times H^{\text {int }}$. Since $\nabla \times V=F_{2}-m H^{\text {int }}$ and $\nu \times V \in L_{t}^{2}\left(\Gamma_{2}\right)$, then $V \in X\left(D, \Gamma_{2}\right)$. Moreover, $V$ satisfies

$$
\nabla \times(\nabla \times V)+m n(x) V=\nabla \times F_{2}
$$

interpreted in the sense of distributions. Now taking the $L^{2}$ scalar product of the first equation of $(3.2 a)$ with a function $\Phi \in H(\operatorname{curl}, D)$, integrating by parts and using the boundary condition $(3.2 b)$, which now takes the form

$$
\nu \times(\nabla \times H)-\nu \times V=f \quad \text { on } \Gamma,
$$

we obtain

$$
\int_{D}[(\nabla \times H) \cdot(\nabla \times \Phi)+H \cdot \Psi] \mathrm{d} x+\int_{\Gamma}(\nu \times V) \cdot \Phi_{\mathrm{T}} \mathrm{d} s=\int_{D} F_{1} \cdot \Phi \mathrm{d} x-\int_{\Gamma} f \cdot \Phi_{\mathrm{T}} \mathrm{d} s .
$$


We now take the $L^{2}$ scalar product of (3.6) with a function $\Psi \in X\left(D, \Gamma_{2}\right)$ and integrate by parts to obtain

$$
\begin{aligned}
\int_{D}\left[\frac{1}{m}(\nabla \times V) \cdot(\nabla \times \Phi)+n V \cdot \Psi\right] \mathrm{d} x-\frac{1}{m} \int_{D} F_{2} \cdot(\nabla \times \Psi) \mathrm{d} x \\
-\frac{1}{m} \int_{\Gamma}(\nabla \times V)_{\mathrm{T}} \cdot(\nu \times \Psi) \mathrm{d} s+\frac{1}{m} \int_{\Gamma} F_{2 T} \cdot(\nu \times \Psi) \mathrm{d} s=0 .
\end{aligned}
$$

From the fact that $\nabla \times V=F_{2}-m H^{\text {int }}$ and the boundary condition $(3.2 d)$, we have

$$
\begin{aligned}
\frac{1}{m} \int_{\Gamma}\left[-(\nabla \times V)_{\mathrm{T}}+F_{2 T}\right] \cdot(\nu \times \Psi) \mathrm{d} s & \\
=\int_{\Gamma} H_{\mathrm{T}} \cdot(\nu \times \Psi) \mathrm{d} s+\frac{\mathrm{i}}{k} \int_{\Gamma_{2}} \eta[\nu \times(\nabla \times H)] \cdot(\nu \times \Psi) \mathrm{d} s & \quad-\int_{\Gamma}(h \times \nu) \cdot(\nu \times \Psi) \mathrm{d} s
\end{aligned}
$$

Combining (3.9) with (3.8) and using (3.2b) we finally obtain

$$
\begin{aligned}
& \int_{D}\left[\frac{1}{m}(\nabla \times V) \cdot(\nabla \times \Psi)+n V \cdot \Psi\right] \mathrm{d} x \\
& \quad+\frac{\mathrm{i}}{k} \int_{\Gamma_{2}} \eta(\nu \times V) \cdot(\nu \times \Psi) \mathrm{d} s+\int_{\Gamma} H_{\mathrm{T}} \cdot(\nu \times \Psi) \mathrm{d} s \\
& \quad=\frac{1}{m} \int_{D} F_{2} \cdot(\nabla \times \Psi) \mathrm{d} x+\int_{\Gamma}(h \times \nu) \cdot(\nu \times \Psi) \mathrm{d} s-\frac{\mathrm{i}}{k} \int_{\Gamma_{2}} \eta f \cdot(\nu \times \Psi) \mathrm{d} s
\end{aligned}
$$

Adding (3.7) and (3.10) shows that $(H, V)$ is a solution of (3.5).

(b) Let $(H, V)$ be a solution of (3.5). It is obvious that $H$ and $V$ satisfy (3.7) and (3.10), respectively. Set

$$
H^{\text {int }}:=-\frac{1}{m} \nabla \times V+\frac{F_{2}}{m} .
$$

By taking sufficiently smooth test functions $\Psi$ in (3.10) we see that $V$ satisfies

$$
\frac{1}{m} \nabla \times(\nabla \times V)+n(x) V=\nabla \times \frac{F_{2}}{m} \quad \text { in } D,
$$

which means that $\nabla \times H^{\text {int }}+n(x) V=0$ in $D$. Therefore, $H^{\text {int }}$ is in $H(\operatorname{curl}, D)$ such that $\nu \times\left. n^{-1}\left(\nabla \times H^{\text {int }}\right)\right|_{\Gamma_{2}} \in L^{2}\left(\Gamma_{2}\right)$ and satisfies the second equation of $(3.2 a)$. By now taking smooth functions $\Phi$ the variational expression (3.7) yields the first equation of $(3.2 a)$. It is easy to verify that the boundary conditions $(3.2 b)-(3.2 d)$ for $H$ and $H^{\text {int }}$ are also satisfied. This ends the proof.

Theorem 3.2. Assume that there exists a constant $\gamma>1$ such that, for $x \in D$,

$$
\operatorname{Re}(\bar{\xi} \cdot n(x) \xi) \geqslant \gamma|\xi|^{2} \quad \forall \xi \in \mathbb{C}^{3} \text { and } \frac{1}{m} \geqslant \gamma .
$$


Then problem (3.5) has a unique solution $(H, V) \in H(\operatorname{curl}, D) \times X\left(D, \Gamma_{2}\right)$. This solution satisfies the a priori estimate

$$
\|H\|_{H(\operatorname{curl}, D)}+\|V\|_{X\left(D, \Gamma_{2}\right)} \leqslant C\left(\left\|F_{1}\right\|_{L^{2}(D)}+\left\|F_{2}\right\|_{L^{2}(D)}+\|f\|_{Y(\Gamma)}+\|h\|_{Y(\Gamma)}\right),
$$

where the constant $C>0$ is independent of $F_{1}, F_{2}, f, h$ and $\gamma$.

Proof. Classical trace theorems and Schwarz's inequality ensure the continuity of the sesquilinear form $\mathcal{A}$ and of the antilinear form $L$ on $H(\operatorname{curl}, D) \times X\left(D, \Gamma_{2}\right)$ as well as the existence of a positive constant $c$ independent of $F_{1}, F_{2}, f$ and $h$ such that

$$
\|L\| \leqslant c\left(\left\|F_{1}\right\|_{L^{2}}+\left\|F_{2}\right\|_{L^{2}}+\|f\|_{Y}+\|h\|_{Y}\right) .
$$

Next we take the real part of $\mathcal{A}$ for $(H, V) \in H(\operatorname{curl}, D) \times X\left(D, \Gamma_{2}\right)$ and use the assumption (3.11) to obtain

$$
\operatorname{Re}(\mathcal{A}(H, V ; \bar{H}, \bar{V})) \geqslant \gamma\|H\|_{H(\operatorname{curl}, D)}^{2}+\|V\|_{H(\operatorname{curl}, D)}^{2}+2 \operatorname{Re}(\langle\bar{H}, V\rangle),
$$

where $\langle\bar{H}, V\rangle$ denotes the duality between $H_{\mathrm{div}}^{-1 / 2}$ and $H_{\text {curl }}^{-1 / 2}$ defined by

$$
\langle\bar{H}, V\rangle:=\int_{\Gamma}(\nu \times V) \cdot \bar{H}_{\mathrm{T}} \mathrm{d} s=\int_{D}[(\nabla \times V) \cdot \bar{H}-(\nabla \times \bar{H}) \cdot V] \mathrm{d} x .
$$

By Schwarz's inequality we have that

$$
|\langle\bar{H}, V\rangle| \leqslant\|H\|_{H(\operatorname{curl}, D)}\|V\|_{H(\operatorname{curl}, D)}
$$

and therefore

$$
\operatorname{Re}(\mathcal{A}(H, V ; \bar{H}, \bar{V})) \geqslant \gamma\|H\|_{H(\operatorname{curl}, D)}^{2}+\|V\|_{H(\operatorname{curl}, D)}^{2}-2\|H\|_{H(\operatorname{curl}, D)}\|V\|_{H(\operatorname{curl}, D)} .
$$

Using the identity

$$
\gamma x^{2}+y^{2}-2 x y=\frac{\gamma+1}{2}\left(x-\frac{2}{\gamma+1} y\right)^{2}+\frac{\gamma-1}{2} x^{2}+\frac{\gamma-1}{\gamma+1} y^{2},
$$

we conclude that

$$
\operatorname{Re}(\mathcal{A}(H, V ; \bar{H}, \bar{V})) \geqslant \frac{\gamma-1}{\gamma+1}\left(\|H\|_{H(\operatorname{curl}, D)}^{2}+\|V\|_{H(\operatorname{curl}, D)}^{2}\right) .
$$

Now taking the imaginary part of $\mathcal{A}$ and using the facts that $\operatorname{Im}(n) \geqslant 0, \eta>0$ and $\eta \in C^{1}\left(\Gamma_{2}\right)$ implies that there exits a positive constant $c$ such that

$$
\operatorname{Im}(\mathcal{A}(H, V ; \bar{H}, \bar{V})) \geqslant c\|\nu \times V\|_{L^{2}\left(\Gamma_{2}\right)} .
$$

Hence we have that

$$
|\mathcal{A}(H, V ; \bar{H}, \bar{V})| \geqslant C_{1}\left(\|H\|_{H(\operatorname{curl}, D)}^{2}+\|V\|_{X\left(D, \Gamma_{2}\right)}^{2}\right)
$$

for some $C_{1}>0$ and thus $\mathcal{A}$ is coercive. The unique determination of $(H, V)$ and the $a$ priori estimate are therefore a direct consequence of the Lax-Milgram lemma applied to $\mathcal{A}$ in $H(\operatorname{curl}, D) \times X\left(D, \Gamma_{2}\right)$ and (3.13). This proves the theorem. 
Theorem 3.3. Under the assumptions of Theorem 3.2, the problem (3.2) has a unique solution $\left(H, H^{\text {int }}\right) \in H(\operatorname{curl}, D) \times H(\operatorname{curl}, D)$, such that $\nu \times\left.(\nabla \times H)\right|_{\Gamma_{2}} \in L^{2}\left(\Gamma_{2}\right)$. This solution satisfies the a priori estimate

$$
\begin{array}{r}
\|H\|_{H(\operatorname{curl}, D)}+\left\|H^{\mathrm{int}}\right\|_{H(\operatorname{curl}, D)}+\|\nu \times(\nabla \times H)\|_{L^{2}\left(\Gamma_{2}\right)} \\
\quad \leqslant 2 C\left(\left\|F_{1}\right\|_{L^{2}(D)}+\left\|F_{2}\right\|_{L^{2}(D)}+\|f\|_{Y(\Gamma)}+\|h\|_{Y(\Gamma)}\right)
\end{array}
$$

where the constant $C>0$ is independent of $F_{1}, F_{2}, f, h$ and $\gamma$.

Proof. It only remains to prove that the uniqueness of the variational problem (3.5) implies the uniqueness of the modified interior transmission problem (3.2). Then the theorem is a consequence of Theorems 3.1 and 3.2.

Consider two solutions $\left(H_{1}, H_{1}^{\text {int }}\right)$ and $\left(H_{2}, H_{2}^{\text {int }}\right)$ to $(3.2)$. Then from Theorem 3.1 $\left(H_{1}, n \nabla \times H_{1}^{\text {int }}\right)$ and $\left(H_{2}, n \nabla \times H_{2}^{\text {int }}\right)$ are two solutions to (3.5), whence $H_{1}=H_{2}$ and $n\left(\nabla \times H_{1}^{\text {int }}\right)=n\left(\nabla \times H_{2}^{\text {int }}\right)$. Since $n^{-1}$ is bounded and $D$ simply connected, the latter means that there exists a function $P \in H^{1}(D)$, uniquely determined up to a real constant, such that $H_{1}^{\text {int }}-H_{2}^{\text {int }}=\nabla P$. The second equation of $(3.2 a)$ yields $m \nabla P=0$ in $D$ and whence $H_{1}^{\text {int }}=H_{2}^{\text {int }}$.

The extra condition on the matrix $n, \operatorname{Re}(\bar{\xi} \cdot n(x) \xi) \geqslant \gamma|\xi|^{2}$ for some $\gamma>1, \forall x \in D$ and $\xi \in \mathbb{C}^{3}$, is not an essential restriction. In particular, it is possible to prove that if $\operatorname{Re}\left(\bar{\xi} \cdot n^{-1} \xi\right) \geqslant \gamma|\xi|^{2}$ for some $\gamma>1$, then there exists a unique solution of the modified transmission problem (3.2). In this case we write a variational formulation for $V:=\nabla \times H$ and $H^{\text {int }}$ in $X\left(D, \Gamma_{2}\right)$ and $H(\operatorname{curl}, D)$, respectively, and follow a similar procedure to that above (see $[\mathbf{2}]$ for the corresponding scalar case). Note also that, since $n$ is a symmetric matrix, $\operatorname{Re}(\bar{\xi} \cdot n \xi)=\bar{\xi} \cdot \operatorname{Re}(n) \xi$.

\section{Uniqueness for the inverse problem}

Now we consider the following inverse problem corresponding to the direct problem of $\S$ 2.1: given, on a large sphere $S_{R}$ of radius $R$ surrounding $\bar{D}$, the scattered fields $\left.E^{\mathrm{s}}\right|_{S_{R}}$ and $\left.H^{\mathrm{s}}\right|_{S_{R}}$ for all incident plane waves $E^{\mathrm{i}}(x)=(\mathrm{i} / k) \nabla \times \nabla \times p \mathrm{e}^{\mathrm{i} k x \cdot d}$ and $H^{\mathrm{i}}(x):=\nabla \times p \mathrm{e}^{\mathrm{i} k x \cdot d}$, $x \in \mathbb{R}^{3}$, with polarization $p \in \mathbb{R}^{3}$ and incident direction $d$ on the unit sphere, find the support $D$ of $n$ (note that $H$ field can be computed from the $E$ field and vice versa). The scattered fields $E^{\mathrm{s}}$ and $H^{\mathrm{s}}$ are the solution to the transmission problem (TP) with the boundary data $f:=\nu \times\left. E^{\mathrm{i}}\right|_{\Gamma}$ and $h:=\nu \times\left. H^{\mathrm{i}}\right|_{\Gamma}$. The main result of this section states that $D$ can be uniquely determined by these data. We note that from Rellich's lemma the scattered fields $\left.E^{\mathrm{s}}\right|_{S_{R}}$ and $\left.H^{\mathrm{s}}\right|_{S_{R}}$ on $S_{R}$ can be uniquely computed by the electric (or magnetic) far-field pattern and conversely [6]. Hence the result is equivalent to the unique determination of $D$ from the electric or magnetic far-field pattern corresponding to all incident directions and all polarizations.

Theorem 4.1. Let the domains $D^{1}$ and $D^{2}$ with the boundaries $\Gamma^{1}$ and $\Gamma^{2}$, respectively, the matrix-valued functions $n_{1}$ and $n_{2}$ and the functions $\eta_{1}$ and $\eta_{2}$ determined on 
the portions $\Gamma_{2}^{1} \subseteq \Gamma^{1}$ and $\Gamma_{2}^{2} \subseteq \Gamma^{2}$, respectively (either $\Gamma_{2}^{1}$ or $\Gamma_{2}^{2}$, or both can possibly be empty sets), satisfy the assumptions of (TP) in $\S \S 2.2$ and 2.3. Moreover, let us assume that either $\bar{\xi} \cdot \operatorname{Re}\left(n_{1}\right) \xi \geqslant \gamma|\xi|^{2}$ or $\bar{\xi} \cdot \operatorname{Re}\left(n_{1}^{-1}\right) \xi \geqslant \gamma|\xi|^{2}$, and either $\bar{\xi} \cdot \operatorname{Re}\left(n_{2}\right) \xi \geqslant \gamma|\xi|^{2}$ or $\bar{\xi} \cdot \operatorname{Re}\left(n_{2}^{-1}\right) \xi \geqslant \gamma|\xi|^{2}$ for some $\gamma>1$. If the scattered fields $\left(E_{1}, H_{1}\right)$ corresponding to the data $D^{1}, n_{1}, \eta_{1}$ and $\left(E_{2}, H_{2}\right)$ corresponding to the data $D^{2}, n_{2}, \eta_{2}$ coincide on a large sphere $S_{R}$ of radius $R$ for all incident plane waves with arbitrary direction $d$ and polarization $p$, then $D^{1} \equiv D^{2}$.

Proof. Consider the scattering of electric dipole fields given by

$$
\left.\begin{array}{l}
E_{\mathrm{e}}^{\mathrm{i}}(x ; z, p)=\frac{\mathrm{i}}{k} \nabla_{x} \times\left(\nabla_{x} \times p \Phi(x, z)\right), \\
H_{\mathrm{e}}^{\mathrm{i}}(x ; z, p)=\nabla_{x} \times p \Phi(x, z),
\end{array}\right\}
$$

due to an electric dipole with polarization $p$ located at $z$, where $\Phi(x, z)$ is the fundamental solution to the Helmholtz equation given by

$$
\Phi(x, z):=\frac{1}{4 \pi} \frac{\mathrm{e}^{\mathrm{i} k|x-z|}}{|x-z|}, \quad x \neq z .
$$

Denote the corresponding scattered waves by $E_{\mathrm{e}}^{\mathrm{s}}(\cdot, z, p)$ and $H_{\mathrm{e}}^{\mathrm{s}}(\cdot, z, p)$. Then, under the assumptions of the theorem, it was shown in [6, Theorem 7.1] that the scattered fields $E_{\mathrm{e}}^{s, 1}, H_{\mathrm{e}}^{s, 1}$ and $E_{\mathrm{e}}^{s, 2}, H_{\mathrm{e}}^{s, 2}$ coincide on $S_{R}$. Note that from Rellich's lemma the scattered fields on $S_{R}$ can be uniquely computed by the far-field patterns and conversely [6].

Now let us assume that $D_{1}$ is not included in $D_{2}$ and let $G$ denote the unbounded connected component of $\mathbb{R}^{3} \backslash\left(\bar{D}^{1} \cap \bar{D}^{2}\right)$. Then there exists a point $z$ such that $z \in \Gamma^{1}$ and $z \notin \Gamma^{2}$. In particular, we have that the points $z_{n}=z+(\epsilon / n) \nu(z)$ lie in $G$ for all $n \in \mathbb{N}$ and $\epsilon$ sufficiently small, where $\nu(z)$ is the outward normal vector to $\Gamma^{1}$ at $z$.

Due to the singular behaviour of $\Phi(x, z)$, it is obvious that $\left\|H_{\mathrm{e}}^{\mathrm{i}}\left(\cdot, z_{n}, \nu(z)\right)\right\|_{H\left(\operatorname{curl}, D^{1}\right)} \rightarrow$ $\infty$ as $n \rightarrow \infty$, where $H_{\mathrm{e}}^{\mathrm{i}}\left(\cdot, z_{n}, \nu(z)\right)$ is the magnetic field of the electric dipole (4.1) with polarization $\nu(z)$. We now consider the incident fields

$$
\left.\begin{array}{l}
H_{n}(x)=\frac{H_{\mathrm{e}}^{\mathrm{i}}\left(x ; z_{n}, \nu(z)\right)}{\left\|H_{\mathrm{e}}^{\mathrm{i}}\left(\cdot ; z_{n}, \nu(z)\right)\right\|_{H\left(\operatorname{curl}, D^{1}\right)}+\left\|\nabla \times H_{\mathrm{e}}^{\mathrm{i}}\left(\cdot ; z_{n}, \nu(z)\right)\right\|_{L^{2}\left(\Gamma_{2}^{1}\right)}}, \\
E_{n}(x)=-\frac{1}{\mathrm{i} k} \nabla \times H_{n}(x)
\end{array}\right\}
$$

for $x \in \bar{D}^{1} \cup \bar{D}^{2}$ and denote by $E_{n}^{j, \mathrm{~s}}, H_{n}^{j, \mathrm{~s}}$ and $E_{n}^{j, \text { int }}, H_{n}^{j, \text { int }}$ the corresponding solutions of (TP) for the domains $D^{j}, j=1,2$. Note that $E_{n}(x)$ is uniformly bounded in $X\left(D^{1}, \Gamma_{2}^{1}\right)$. Next we want to show that the sequence $H_{n}$ is uniformly bounded in $H^{1}\left(D^{1}\right)$ and consequently in $X\left(D^{1}, \Gamma_{2}^{1}\right)$. Theorem 2.3 will then show that the corresponding scattered fields and interior fields are uniformly bounded in their respective norms. To this end with the help of a cut-off function $\chi$ supported in $B_{2 \epsilon}(z)$ and $\chi=1$ in $B_{\epsilon}(z)$, we first write

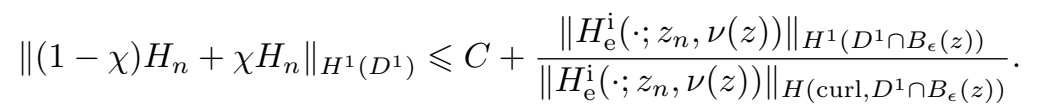


Simple computations show that

$$
\begin{aligned}
\left\|H_{\mathrm{e}}^{\mathrm{i}}\left(\cdot ; z_{n}, \nu(z)\right)\right\|_{H^{1}\left(D^{1} \cap B_{\epsilon}(z)\right)}^{2}= & \left\|\nabla_{x} \Phi\left(x, z_{n}\right) \times \nu(z)\right\|_{L^{2}\left(D^{1} \cap B_{\epsilon}(z)\right)}^{2} \\
& +\left\|\nabla_{x} \nabla_{x} \Phi\left(x, z_{n}\right) \times \nu(z)\right\|_{L^{2}\left(D^{1} \cap B_{\epsilon}(z)\right)}^{2} \\
= & \frac{1}{\left|z_{n}-z\right|^{3}}\left[A_{1}+O\left(\left|z_{n}-z\right|\right)\right]
\end{aligned}
$$

and

$$
\begin{aligned}
\left\|H_{\mathrm{e}}^{\mathrm{i}}\left(\cdot ; z_{n}, \nu(z)\right)\right\|_{H\left(\operatorname{curl}, D^{1} \cap B_{\epsilon}(z)\right)=}^{2}= & \left\|\nabla_{x} \Phi\left(x, z_{n}\right) \times \nu(z)\right\|_{L^{2}\left(D^{1} \cap B_{\epsilon}(z)\right)}^{2} \\
& +\left\|k^{2} \Phi\left(x, z_{n}\right) \nu(z)+\nabla_{x} \nabla_{x} \Phi\left(x, z_{n}\right) \cdot \nu(z)\right\|_{L^{2}\left(D^{1} \cap B_{\epsilon}(z)\right)}^{2} \\
= & \frac{1}{\left|z_{n}-z\right|^{3}}\left[A_{2}+O\left(\left|z_{n}-z\right|\right)\right] .
\end{aligned}
$$

Furthermore, a straightforward but long computation shows that

$$
A_{2}=2 \pi \int_{\pi / 2}^{\pi} \int_{0}^{\infty} \frac{t^{2}\left(3 \cos ^{2} \theta+1\right) \sin \theta}{\left(t^{2}+1-2 t \cos \theta\right)^{3}} \mathrm{~d} t \mathrm{~d} \theta>0
$$

whence (4.3) is uniformly bounded for $n \in \mathbb{N}$.

Now let $B_{\epsilon}(z)$ be a ball of radius $\epsilon>0$ centred at $z$. Since $E_{\mathrm{e}}\left(\cdot, z_{n}, \nu(z)\right)$ and $H_{\mathrm{e}}\left(\cdot, z_{n}, \nu(z)\right)$ together with their derivatives are uniformly bounded in every compact subset of $\mathbb{R}^{3} \backslash B_{2 \epsilon}(z)$, we have that

$$
\lim _{n \rightarrow \infty}\left\|E_{n}\right\|_{H^{1}\left(D^{2}\right)}=\lim _{n \rightarrow \infty}\left\|H_{n}\right\|_{H^{1}\left(D^{2}\right)}=0
$$

whence

$$
\lim _{n \rightarrow \infty}\left\|E_{n}^{2, \mathrm{~s}}\right\|_{H^{1}\left(B_{R} \cap G\right)}=\lim _{n \rightarrow \infty}\left\|H_{n}^{2, \mathrm{~s}}\right\|_{H^{1}\left(B_{R} \cap G\right)}=0
$$

from the estimates $(2.32),(2.41),(2.43)$ applied to the scattered field corresponding to $D^{2}$. But $\left.\hat{x} \cdot H_{n}^{1, \mathrm{~s}}\right|_{S_{R}}=\left.\hat{x} \cdot H_{n}^{2, \mathrm{~s}}\right|_{S_{R}}$ and therefore by the uniqueness of the exterior Maxwell problem outside $B_{R}$ and unique continuation we conclude that

$$
\begin{aligned}
& \lim _{n \rightarrow \infty}\left\|E_{n}^{1, \mathrm{~s}}\right\|_{H^{1}\left(B_{R} \cap G\right)}=\lim _{n \rightarrow \infty}\left\|E_{n}^{2, \mathrm{~s}}\right\|_{H^{1}\left(B_{R} \cap G\right)}=0, \\
& \lim _{n \rightarrow \infty}\left\|H_{n}^{1, \mathrm{~s}}\right\|_{H^{1}\left(B_{R} \cap G\right)}=\lim _{n \rightarrow \infty}\left\|H_{n}^{2, \mathrm{~s}}\right\|_{H^{1}\left(B_{R} \cap G\right)}=0 .
\end{aligned}
$$

Hence from trace theorems and the help of a cut-off function $\chi \in C_{0}^{\infty}\left(B_{\epsilon^{\prime}}(z)\right)$, where $\epsilon^{\prime}>0$ is small enough to ensure that $B_{\epsilon^{\prime}}(z) \cap D^{1}=B_{\epsilon^{\prime}}(z) \cap \mathbb{Z}$, we conclude that

$$
\lim _{n \rightarrow \infty}\left\|\nu \times \chi H_{n}^{1, \mathrm{~s}}\right\|_{Y\left(\Gamma^{1}\right)}=\lim _{n \rightarrow \infty}\left\|\nu \times\left(\nabla \times \chi H_{n}^{1, \mathrm{~s}}\right)\right\|_{Y\left(\Gamma^{1}\right)}=0 .
$$

In the exterior of $B_{2 \epsilon}(z)$ the $H^{1}\left(B_{R} \backslash B_{2 \epsilon}(z)\right)$ norm of $E_{n}$ and $H_{n}$ remain uniformly bounded and therefore from Remark $2.7(1-\chi) E_{n}^{1, \mathrm{~s}},(1-\chi) H_{n}^{1, \mathrm{~s}}$ are also uniformly bounded in $H^{1}\left(\left(B_{R} \cap D_{\mathrm{e}}^{1}\right) \backslash B_{2 \epsilon}(z)\right)$. Using the compact imbedding of $H^{1}\left(B_{R} \cap D_{\mathrm{e}}^{1}\right)$ into 
$H^{(1-\tau)}\left(B_{R} \cap D_{\mathrm{e}}^{1}\right)$ for $0<\tau \leqslant 1$, we can now select a $X\left(B_{R} \cap D_{\mathrm{e}}^{1}\right)$ convergent subsequence $(1-\chi) H_{n_{j}}^{1, \mathrm{~s}}$ from $(1-\chi) H_{n}^{1, \mathrm{~s}}$. Hence, $\nu \times(1-\chi) H_{n_{j}}^{1, \mathrm{~s}}$ and $\nu \times\left[\nabla \times(1-\chi) H_{n_{j}}^{1, \mathrm{~s}}\right]$ are convergent in $Y\left(\Gamma^{1}\right)$ as well. Combining this fact with (4.4) we have that the sequences

$$
\nu \times H_{n_{j}}^{1, \mathrm{~s}} \text { and } \quad \nu \times\left(\nabla \times H_{n_{j}}^{1, \mathrm{~s}}\right)
$$

converge in the trace space $Y\left(\Gamma^{1}\right)$.

The estimate (2.41) shows that $H_{n}^{1, \text { int }}$ is uniformly bounded in $H^{1}\left(D^{1}\right)$ because $H_{n}$ is uniformly bounded in $H^{1}\left(D^{1}\right)$ and consequently the boundary data are uniformly bounded in the required trace spaces. Since $H_{n_{j}}$ and $H_{n_{j}}^{1, \text { int }}$ is a solution of the interior transmission problem (3.1) in $D^{1}$ with boundary data produced by the exterior field $H_{n_{j}}^{1, \mathrm{~s}}$ we have that $H_{n_{j}}$ and $H_{n_{j}}^{1, \text { int }}$ also solve the modified interior transmission problem (3.2) with $F_{1}:=H_{n_{j}}$ and $F_{2}:=H_{n_{j}}^{1 \text {,int }}$ and boundary data $f:=\nu \times\left(\nabla \times H_{n_{j}}^{1, \mathrm{~s}}\right), h:=\nu \times H_{n_{j}}^{1, \mathrm{~s}}$. By using the compact imbedding of $H^{1}\left(D^{1}\right)$ in $L^{2}\left(D^{1}\right)$ we can select from $H_{n_{j}}$ and $H_{n_{j}}^{1, \mathrm{~s}}$ convergent subsequences in $L^{2}\left(D^{1}\right)$, which we again denote by $H_{n_{j}}$ and $H_{n_{j}}^{1, \mathrm{~s}}$. The estimate (3.14) in Theorem 3.3 now gives that $H_{n_{j}}$ converges with respect to the norm $H\left(\operatorname{curl}, D^{1}\right)$ to $H_{0} \in H\left(\operatorname{curl}, D^{1}\right)$ and moreover $\nabla \times\left. H_{n_{j}}\right|_{\Gamma_{2}^{1}}$ converges to $\nabla \times\left. H_{0}\right|_{\Gamma_{2}^{1}}$ with respect to $L_{t}^{2}\left(\Gamma_{2}^{1}\right)$. Obviously, $H_{0}$ satisfies $\nabla \times\left(\nabla \times H_{0}\right)-k^{2} H_{0}=0$ in the weak sense. But $\left.H_{0}\right|_{D^{1} \backslash B_{2 \epsilon}(z)}=0$ since the function $H_{n_{j}}$ converges uniformly to zero outside the ball $B_{2 \epsilon}$. Therefore, $H_{0}$ must be equivalent to zero in all of $D^{1}$. But this contradicts the fact that $\left\|H_{n}\right\|_{H\left(\operatorname{curl}, D^{1}\right)}+\left\|\nabla \times H_{n}\right\|_{L_{t}^{2}\left(\Gamma_{2}^{1}\right)}=1$ for all $n \in \mathbb{N}$.

Since we can derive the same contradiction for the assumption that $D^{2}$ is not included in $D^{1}$, we have proved that $D^{1}=D^{2}$.

Acknowledgements. This research was supported in part by grants from the Air Force Office of Scientific Research.

\section{References}

1. T. S. Angell AND A. KIRSCH, The conductive boundary condition for Maxwell's equations, SIAM J. Appl. Math. 52 (1992), 1597-1610.

2. F. CAKONI AND H. HAdDAR, The linear sampling method for anisotropic media, Part 2, Preprint 2001/26, MSRI Berkeley, CA (2001).

3. F. CAKoni, D. Colton And H. Haddar, The linear sampling method for anisotropic media, J. Comput. Appl. Math. 146 (2002), 285-299.

4. F. CAKoni, D. Colton And P. Monk, The electromagnetic inverse scattering problem for partially coated Lipschitz domains, submitted.

5. S. CoArsi, P. Fernandes and M. Raffetto, On the convergence of Galerkin finite element approximations of electromagnetic eigenproblems, SIAM J. Numer. Analysis 38 (2000), 1129-1145.

6. D. Colton And R. KRess, Inverse acoustic and electromagnetic scattering theory, 2nd edn (Springer, 1998).

7. D. COLTON AND P. Monk, Herglotz wave functions in inverse electromagnetic scattering theory, in Computational methods in wave propagation (ed. M. Ainsworth) (Springer, 2003), in press. 
8. D. COLTON AND L. PÄIVÄRINTA, The uniqueness of a solution to an inverse scattering problem for electromagnetic waves, Arch. Ration. Mech. Analysis 119 (1992), 59-70.

9. M. Costabel, A remark on the regularity of solutions of Maxwell's equations on Lipschitz domains, Math. Meth. Appl. Sci. 12 (1990), 365-368.

10. P. HÄHNER, A uniqueness theorem for a transmission problem in inverse electromagnetic scattering, Inverse Problems 9 (1993), 667-678.

11. P. Ḧ̈HnER, On the uniqueness of the shape of a penetrable, anisotropic obstacle, $J$. Comput. Appl. Math. 116 (2000), 167-180.

12. P. Ḧ̈HNER, Electromagnetic wave scattering: theory, in Scattering (ed. R. Pike and P.Sabatier), pp. 211-229 (Academic, 2002).

13. F. Hettlich, Uniqueness of the inverse conductive scattering problem for time-harmonic electromagnetic waves, SIAM J. Appl. Math. 2 (1996), 588-601.

14. A. KIRSCH AND R. KRESS, Uniqueness in inverse obstacle scattering, Inverse Problems 9 (1993), 285-299.

15. A. KIRSCH AND P. MonK, A finite element/spectral method for approximating the time harmonic Maxwell system in $R^{3}$, SIAM J. Appl. Math. 55 (1995), 1324-1344 (Corrigendum: SIAM J. Appl. Math. 58 (1998), 2024-2028).

16. P. MONK, Finite element methods for Maxwell's equations (Oxford University Press, 2002).

17. J. C. NÉDÉLEC, Acoustic and electromagnetic equations. Integral representations for harmonic problems (Springer, 2001).

18. T. ÔKAJI, Strong unique continuation property for time harmonic Maxwell equations, $J$. Math. Soc. Jpn 54 (2002), 89-122.

19. P. Ola AND E. Somersalo, Electromagnetic inverse problems and generalized Sommerfeld potentials, SIAM J. Appl. Math. 56 (1996), 1129-1145.

20. P. Ola, L. PÄIVÄrinta AND E. Somersalo, An inverse boundary value problem in electrodynamics, Duke Math. J. 70 (1993), 617-653.

21. B. P. Rynne And B. D. SleEman, The interior transmission problem and inverse scattering from inhomogeneous media, SIAM J. Math. Analysis 22 (1991), 1755-1762.

22. G. Uhlmann, Inverse scattering in anisotropic media, in Surveys on solution methods for inverse problems (ed. D. Colton et al.) (Springer, 2000), pp. 235-251. 